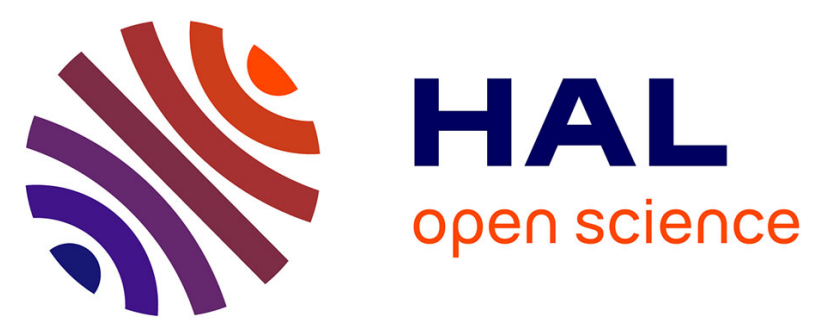

\title{
Cinq mesures romaines graduées en bois de la colonie romaine d'Arausio/Orange (Vaucluse) : étude de leurs applications à l'artisanat du petit mobilier
}

Philippe Prévot

\section{To cite this version:}

Philippe Prévot. Cinq mesures romaines graduées en bois de la colonie romaine d'Arausio/Orange (Vaucluse) : étude de leurs applications à l'artisanat du petit mobilier. Gallia - Archéologie de la France antique, 2008, 65, pp.327-353. 10.3406/galia.2008.3347 . hal-01899177

\author{
HAL Id: hal-01899177 \\ https://hal.science/hal-01899177
}

Submitted on 19 Dec 2019

HAL is a multi-disciplinary open access archive for the deposit and dissemination of scientific research documents, whether they are published or not. The documents may come from teaching and research institutions in France or abroad, or from public or private research centers.
L'archive ouverte pluridisciplinaire HAL, est destinée au dépôt et à la diffusion de documents scientifiques de niveau recherche, publiés ou non, émanant des établissements d'enseignement et de recherche français ou étrangers, des laboratoires publics ou privés.

\section{(1) (1) $\$$}

Distributed under a Creative Commons Attribution - NonCommercial - NoDerivatives 44.0 


\title{
CINQ MESURES ROMAINES GRADUÉES EN BOIS DE LA COLONIE ROMAINE D'ARAUSIO/ORANGE (VAUCLUSE)
}

\author{
Étude de leurs applications à l'artisanat du petit mobilier
}

\author{
Philippe PRÉVOT*
}

Mots-clés. Gallo-romain, métrologie, artisanat, instruments de mesure, industrie osseuse, atelier, romanisation, techniques, outils.

Résumé. La fouille menée sur le site dit de la Résorption de l'habitat insalubre de Saint-Florent à Orange/Arausio a permis la découverte d'une série de réglettes graduées en bois. Au-delà du caractère exceptionnel, en Gaule, de ce type d'instruments de mesure, il convient d'analyser les conséquences des concordances métrologiques qui existent avec le pied (pes) romain. En effet, il semble que ces instruments participent à la romanisation par l'adoption du système métrologique dans la colonie romaine d'Arausio. La taille réduite de ces mesures et la précision de leurs graduations laissent supposer qu'elles ont été utilisées à des fins artisanales pour la fabrication de petits objets. Nous poserons la question de leur possible emploi, entre autres, par les artisans de l'os. Ainsi, par une étude des caractères métrologiques de certaines de ces productions, nous verrons de quelle façon l'utilisation de ces règles contribue à la normalisation du travail artisanal.

Key-words. Gallo-Roman, metrology, craft, measuring devices, bone-craft, workshop, Romanization, technique, tools.

Abstract. During the excavation in the site named la Résorption de l'habitat insalubre de Saint-Florent at Orange/Arausio, a series of wooden graduated rulers have been discovered. Outside the fact that this type of measuring instruments are exceptional in Gaul, we have to define the results of the metrological similarities with the Roman foot (pes). It seems that these instruments are part of Romanization by the adoption of the system of metrology in the colonia of Arausio. From the small size of these rulers and their precise graduations we can imagine an utilization relating to crafts, for productions of small items. We shall also put the question of their utilization by bone-workers for example. So the study of the metrological features of some of these products will show how the use of these rulers is a contribution to the normalisation of work.

Translation: Isabelle FAUDUET

Schlüsselwörter. Gallo-römisch, Metrologie, Handwerk, Messinstrumente, Knochenschnitzerei, Werkstatt, Romanisierung, Techniken, Werkzeuge.

Zusammenfassung. Bei der Grabung in der Résorption de l'habitat insalubre de Saint-Florent in Orange/Arausio konnte eine Reihe von graduierten hölzernen Maßstäben sichergestellt werden. Abgesehen von dem außergewöhnlichen Charakter dieser Art Messinstrument in Gallien empfiehlt es sich die Konsequenzen zu analysieren, die sich aus den metrologischen Entsprechungen der Einteilungen mit dem römischen Fuß (pes) ergeben. Durch die Annahme dieses metrischen Systems in der römischen Kolonie von Orange/Arausio scheinen die Instrumente in der Tat zur Romanisierung beigetragen zu haben.

Das kleine Format dieser Messgeräte und die Präzision ihrer Einteilungen lassen vermuten, dass sie der Herstellung kleiner Gegenstände im handwerklichen Bereich dienten. Wir stellen die Frage ihrer möglichen Verwendung u.a. durch Knochenschnitzer. So werden wir anhand einer Untersuchung der metrologischen Eigenschaften einiger dieser Gegenstände erkennen, wie die Benutzung dieser Messgeräte zur Standardisierung der handwerklichen Tätigkeit beigetragen hat.

Übersetzung: Isa ODENHARDT-DONVEZ

\footnotetext{
* IRAA, UMR 6222 du CNRS, Maison méditerranéenne des sciences de l’homme, 5 rue du Château-de-l'Horloge, BP 647, F-13094 Aix-enProvence Cedex 2. Courriel : philippeprevot@hotmail.com
} 
En Gaule, l'usage d'étalons de mesures est attesté à l'époque romaine (Héron de Villefosse, 1902 ; Béal, 1983, p. 357 ; Feugère, 1983 et 1995, p. 151). La diffusion de différents types d'instruments de mesure participant, de cette manière, à la romanisation par l'étalonnage du système du pied romain (pes monetalis) ${ }^{1}$ (Hultsch, 1882 ; Héron de Villefosse, 1907). Il semble, donc, que différents gabarits de mesures aient été reproduits en fonction du type de travail à effectuer et de son échelle. Les découvertes de réglettes (regulae) de deux pieds (pes) en bois ${ }^{2}$ (Fellmann, 1988, p. 200), de pieds en bronze ${ }^{3}$ (Hussong, 1938 ; Rottländer, 1972 et 1991) et de pieds pliants en bronze et en os (Héron de Villefosse, 1902 et 1907 ; Béal, 1983 ; Feugère, 1983), subdivisées en multiples du pied ${ }^{4}$, témoignent de leur utilisation, comme le suggère $M$. Feugère pour des activités artisanales de précision (Feugère, 1995).

Les instruments de mesure non pliants, quant à eux, sont moins connus. En effet, seuls deux exemplaires d'un demi-pied en bronze, étalonnés sur le pes monetalis, sont répertoriés en Gaule. Il s'agit des mesures de Besançon (Doubs), trouvées dans un contexte stratigraphique des années 120-40 av. J.-C. (Feugère, 1992) et de Chalon-surSaône (Saône-et-Loire) ${ }^{5}$ (Feugère, 1995). Ces mesures sont divisées en paumes (palmi), pouces (unciae), demipouces, doigts (digiti) et demi-doigts. Un exemplaire de même type, en bronze, est connu à Mâcon (Saône-etLoire) (Barthélemy, Dubois, 2007). Il comporte certaines subdivisions du pied et semble être étalonné sur le pes drusianus $^{6}$ (tabl. I), plus connu en architecture et dans

1. Bien que plusieurs types de pieds aient été en usage et aient coexisté durant la période romaine, le pied théorique romain reste le pes monetalis, égal à $295,70 \mathrm{~mm}$.

2. Réglette en bois d'if provenant du dépotoir du camp légionnaire de Vindonissa, Suisse.

3. Une mesure en bronze, qui semble en partie étalonnée sur le pes drusianus, a été en outre trouvée à Xanten, en Allemagne (Rottländer, 1994).

4. En effet, les pieds pliants mesurent, une fois repliés, un demi-pied. De plus, ils comportent des subdivisions en pouces et doigts (Héron de Villefosse, 1907, fig. 5603 et 5604 ; Béal, 1983, pl. LX ; Gostenčnik, 1998, p. 93, fig. 3).

5. Ces éléments isolés étaient peut-être insérés dans un dispositif plus complexe (Gostenčnik, 1998, p. 102, fig. 9).

6. Le pes drusianus semble avoir été en usage dès la fin de la République, comme l'atteste désormais la découverte de Mâcon, et pas seulement durant l'époque trajane et pendant le II ${ }^{\mathrm{e}}$ s. apr. J.-C. (Anderson, 2001, p. 72). C'est Hygin qui en fait mention (De condicionibus agrorum, 123, 9-10) : ce système de mesure aurait été utilisé par les Tungri (Germanie inférieure) au début du $\mathrm{II}^{\mathrm{e}}$ s. apr. J.-C. (Kidson, 1990, p. 81-82). Le pes drusianus est égal à $332,70 \mathrm{~mm}$, et Hygin précise qu'il est $1 / 8^{\mathrm{e}}$ plus grand que le pes monetalis, soit un digitus drusianus égal à $18,50 \mathrm{~mm}$. Cette unité de mesure semble avoir été en usage dans certains camps l'élaboration de plans que pour l'artisanat (Duncan-Jones, 1980 ; Millett, 1982 ; Bridger, 1984 ; Kidson, 1990 ; Anderson, 2001). Les instruments de ce type sont morphologiquement adaptés à la mesure ou à la fabrication de petits objets.

L'utilisation de ce type de réglettes est notamment attestée en Italie. En effet, certains de ces instruments sont figurés sur des stèles funéraires. L'étude entreprise par G. Zimmer sur les représentations des métiers et de leurs outils vient nous éclairer sur l'usage de ces mesures dans certains types d'artisanats. C'est le cas de la menuiserie $^{7}$ (Zimmer, 1982, p. 142, $\mathrm{n}^{\circ} 61$ ), de la charpenterie de marine ${ }^{8}$ (op. cit., 1982, p. 146, $\mathrm{n}^{\circ} 65$ ) et du travail du bois en général ${ }^{9}$ (op. cit., 1982, p. 150, nº 70). D’autres réglettes sont sculptées, quant à elles, sur des stèles dont la profession des défunts reste indéterminée (op. cit., 1982, p. $165, \mathrm{n}^{\circ} 88^{10}$; p. $165-166, \mathrm{n}^{\circ} 89^{11}$; p. $166, \mathrm{n}^{\text {o }} 90^{12}$; p. $168-169, \mathrm{n}^{\text {o }} 92^{13}$ ). Dans tous les cas, il semble que ces instruments n'excèdent pas les 2 pieds et qu'ils comportent des graduations principalement étalonnées sur le pouce. Ces mesures restent toutefois plus grandes et moins précises que celles d'un demi-pied connues par les objets eux-mêmes.

En 1992, cinq mesures en bois ${ }^{14}$ de deux gabarits différents ont été découvertes dans la domus E2 (état II) du site dit de la Résorption de l'habitat insalubre (R.H.I.) de Saint-Florent à Orange (Prévot, 2004a). Ces réglettes, trouvées dans le puits de la cour no 2 de la maison, y ont été

militaires romains (comme dans celui de Lauriacum en Autriche, situé dans la province de Norique, au III ${ }^{\mathrm{e}}$ s. apr. J.-C.). Elle paraît avoir servi, de plus, à l'élaboration de plans urbains et de monuments publics, comme à Xanten (Allemagne), en Germanie inférieure, par exemple (Bridger, 1984).

7. La réglette, sculptée sur une stèle provenant de Priolo (Sicile), ne comporte visiblement pas de graduations. Toutefois, l'archet et le trépan, qui sont représentés à ses côtés, nous donnent une indication d'échelle.

8. Sur une stèle funéraire, trouvée à Ostie (Italie), figure une réglette subdivisée transversalement en 16 demi-pouces ou demi-doigts et 5 pouces ou doigts.

9. Il s'agit d'une mesure de deux pieds figurée sur une stèle découverte à Aquilée (Italie) ; elle est subdivisée transversalement en 24 pouces.

10. Cette mesure, gravée sur une stèle provenant de la villa Pedriali (Italie), ne comporte pas de subdivisions visibles.

11. La mesure, sculptée sur une stèle trouvée à Rome (Italie), comporte des subdivisions transversales.

12. Cette stèle a été trouvée à Pise (Italie). On peut distinguer que la réglette est divisée en 18 graduations transversales. Il est probable qu'il s'agisse d'une mesure d'un pied et demi, subdivisée en 18 pouces.

13. La réglette, représentée sur une stèle trouvée à Rome (Italie), ne comporte pas de subdivisions.

14. Il s'agit des mesures $n^{\text {os }} 1$ (n $n^{\circ}$ 95.1.406), 2 ( $n^{\circ}$ 95.1.407 A), 3 $\left(\mathrm{n}^{\circ}\right.$ 95.1.407 B/C), $4\left(\mathrm{n}^{\circ}\right.$ 95.1.407 D) et $5\left(\mathrm{n}^{\circ}\right.$ 95.1.407 E). La numérotation utilisée est celle du musée archéologique d'Orange. 
Tabl. I - Mesures théoriques $d u$ pes drusianus et des subdivisions du semi-pes : *, mesure attestée par Hygin.

\begin{tabular}{|c|c|c|c|c|c|c|c|}
\cline { 2 - 8 } \multicolumn{1}{l|}{} & Pes & Semi-pes & Palmus & Uncia & Semi-uncia & Digitus & Semi-digitus \\
\hline $\mathbf{1}$ & $332,7^{*}$ & 166,4 & 83,2 & 27,7 & 13,9 & 20,8 & 10,4 \\
\hline $\mathbf{2}$ & & 332,7 & 166,4 & 55,5 & 27,7 & 41,6 & 20,8 \\
\hline $\mathbf{3}$ & & & & 83,2 & 41,6 & 62,4 & 31,2 \\
\hline $\mathbf{4}$ & & & & 110,9 & 55,5 & 83,2 & 41,6 \\
\hline $\mathbf{5}$ & & & & 138,6 & 69,3 & 104 & 52 \\
\hline $\mathbf{6}$ & & & & 166,4 & 83,2 & 124,8 & 62,4 \\
\hline $\mathbf{7}$ & & & & & 97 & 145,6 & 72,8 \\
\hline $\mathbf{8}$ & & & & & 110,9 & 166,4 & 83,2 \\
\hline $\mathbf{9}$ & & & & & 124,8 & & 93,6 \\
\hline $\mathbf{1 0}$ & & & & & 138,6 & & 104 \\
\hline $\mathbf{1 1}$ & & & & & 152,5 & & 114,4 \\
\hline $\mathbf{1 2}$ & & & & & 166,4 & & 124,8 \\
\hline $\mathbf{1 3}$ & & & & & & & 135,2 \\
\hline $\mathbf{1 4}$ & & & & & & & 145,6 \\
\hline $\mathbf{1 5}$ & & & & & & & 156 \\
\hline $\mathbf{1 6}$ & & & & & & & 166,4 \\
\hline
\end{tabular}

conservées grâce à l'humidité ${ }^{15}$. Il s'agit d'exemplaires d'un demi-pied en buis (Buxus sempervirens) ${ }^{16}$ comportant toutes les subdivisions connues du pied romain. Aucun exemplaire de ce type de mesure n'ayant été trouvé à ce jour, nous en présentons une étude et une analyse détaillées.

Ainsi, dans un premier temps, nous décrirons les mesures orangeoises et tenterons de les replacer, plus largement, dans le système métrologique romain et le processus de la romanisation. Nous poserons la question de leur fabrication et de leur utilisation en essayant de rattacher cette réflexion à leur contexte archéologique.

Si aucune réglette d'un demi-pied n'a pu être associée à des types d'artisanats particuliers ${ }^{17}$, la précision de leurs subdivisions laisse penser à la fabrication de petits objets en bois, en bronze, en os, en bois de cerf et en ivoire. Toutefois, l'identification et l'étude métrologique sur des objets finis ne semblent être réalisables qu'à partir des matières dures animales (os, ivoire et bois de cerf). En effet, la décou-

15. Ce lot de mesures en bois a été immédiatement traité pour éviter leur dégradation au cours de leur dessiccation.

16. Le buis est longtemps resté l'essence favorite des artisans, apprécié pour sa robustesse et la finesse avec laquelle on pouvait le travailler. Dans la littérature classique, certains auteurs désignent les objets en buis par le mot buxum, qui signifie le buis et notamment les peignes (pecten) (Daremberg et al. dir., Dictionnaire des Antiquités grecques et romaines, s.v. «Buxum », I, 1, p. 756).

17. Hormis le travail du bois pour des dimensions supérieures à un pied. verte d'objets en bois reste rare et la dessiccation conduit à la déformation de la plupart d'entre eux. Dans le cas des objets en bronze, les mesures sont compliquées à retrouver, puisque les objets peuvent être repris et éventuellement abrasés après la fonte.

Nous tenterons alors, et dans un second temps, de cerner les applications métrologiques qui peuvent être faites à l'artisanat de l'os. Nous nous interrogerons donc, à partir des productions de tabletterie orangeoises, sur la possible utilisation d'instruments de mesure de ce type à des fins normalisatrices. Dans un travail récent, nous avons posé la question de la normalisation du travail en tabletterie galloromaine (Prévot, 2004b). En effet, il semble que certains types de déchets de travail et certains types d'objets présentent des dimensions globalement similaires. Ainsi, nous tenterons de déterminer de quelle façon la normalisation des objets en os de la colonie romaine d'Orange est effectuée et si elle s'inscrit dans un souci métrologique ou non.

\section{ÉTAT II DE LA MAISON E2 À SAINT-FLORENT}

Le quartier résidentiel de Saint-Florent est actuellement la plus ancienne occupation romaine reconnue à Orange (fig. 1). En effet, les vestiges archéologiques attestent d'un aménagement contemporain des années 20-10 av. J.-C., soit environ une quinzaine d'années après la fondation théorique de la colonie ${ }^{18}$, datée de 35 av. J.-C.

La fouille, réalisée sur une surface de $3500 \mathrm{~m}^{2}$, a été successivement menée sous la direction de M.-E. Bellet, J.-M. Mignon et A. Hasler, entre 1988 et 1993. Elle a été entreprise dans le cadre de l'opération dite de Résorption de l'habitat insalubre.

Le site, placé entre le centre monumental romain et le rempart est de la ville antique, compte vingt-cinq maisons ou parties de maisons, issues de deux campagnes d'urbanisation successives. La relative homogénéité repérée dans les périodes d'occupation et d'abandon des maisons a permis de dégager deux états.

Le premier état compte quatorze maisons réparties sur les îlots septentrional et méridional. L'installation des premières maisons a pu être datée autour de 15 av. J.-C. ${ }^{19}$

18. Le site devient la Colonia Firma Julia Secundanorum Arausio (Faure et al., 1999).

19. Les premières hypothèses dataient l'installation des années 10 av. J.-C., mais à la suite d'un récent travail universitaire qui a repoussé cette date d'environ cinq années, nous adopterons désormais la date de 15 av. J.-C. (Roumegous, 2003). 


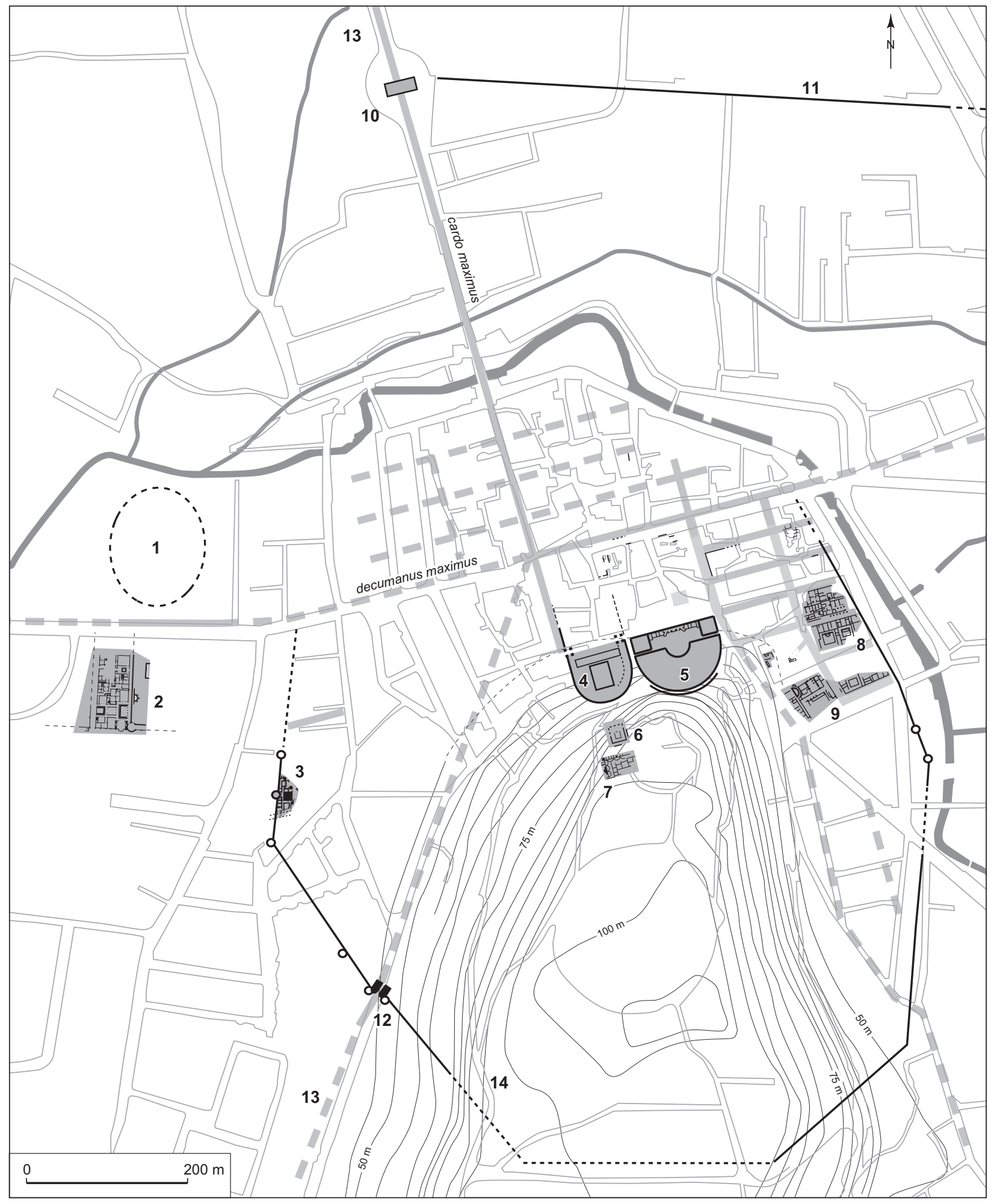

\begin{tabular}{|lllll|}
\hline & voies et rues & 1. Amphitéâtre & 6. Temple (?) & 11. Aqueduc \\
rempart & 2. Site de la Brunette & 7. "Capitole » & 12. Porte sud-ouest \\
& 3. Site du Mas des Thermes & 8. Site de Saint-Florent & 13. Voie d'Agrippa \\
& 4. Site de l'Hémicycle (temple et forum) & 9. Site du cours Pourtoules & 14. Atelier du travail de l'os du \\
5. Théâtre & 10. Arc de Triomphe & chemin de la Maguelonne \\
\hline
\end{tabular}

Fig. 1 - Localisation des principaux sites archéologiques dans Orange antique

(DAO : P. Prévot d'après fond de carte C. Allinne et données archéologiques : J.-M. Mignon, SADV). 
et l'abandon du site semble s'effectuer aux alentours des années 20 apr. J.-C. Selon J.-M. Mignon, la phase d'abandon semble être due à une inondation de la Meyne qui aurait provoqué l'effondrement des murs en adobes et la destruction des maisons (Mignon, 1996a, p. 219).

Le second état présente, lui, dix maisons situées, comme pour le premier, sur les deux îlots (fig. 2). Cet état a pu être daté archéologiquement entre 30-40 apr. J.-C. et le début du III $^{\mathrm{e}}$ s. apr. J.-C. La phase d'abandon semble avoir été suivie de vandalismes importants, comme en témoignent, notamment, les éléments mobiliers jetés dans les puits. La fouille a permis de voir que l'état II respectait généralement la trame urbaine préexistante. Toutefois, un regroupement de certaines parcelles de l'état I a été effectué au cours de l'état II (Mignon, 1996b, p. 236).

Les cinq mesures en bois d'un demi-pied proviennent du puits de la cour $\mathrm{n}^{\mathrm{o}} 2$ de la domus E2 (fig. 3). Elles sont datées archéologiquement de la phase d'abandon de l'état II, soit entre la seconde moitié du $\mathrm{II}^{\mathrm{e}} \mathrm{s}$. et le début du III ${ }^{\mathrm{e}} \mathrm{s}$. apr. J.-C. Les sols d'occupation et les niveaux d'abandon/ destruction, très mal conservés, n'ont pas permis de préciser leur datation: elle n'a été réalisée que par analogie aux autres maisons dont les observations archéologiques montrent qu'elles ont connu des phases chronologiques proches (Mignon, 1996b, p. 274-275).

La maison E2 s'inscrit dans une parcelle de l'îlot septentrional qui jouxte une rue de direction nord-sud (Mignon, 1996b, p. 274-275). Le bâti se compose de deux ensembles de bâtiments distincts compris dans une surface de $226 \mathrm{~m}^{2}$. À l'est, la résidence est implantée sur l'ancienne parcelle E2 et, à l'ouest, les locaux utilitaires sont implantés sur l'ancienne parcelle E3. La résidence comprend un bâtiment principal et un local, disposé au sud, sans doute ultérieurement assimilable à une annexe du bâtiment principal.

Les locaux utilitaires se composent de trois ailes qui enserrent une cour. Il n'est pas improbable que la première pièce, implantée en bordure de la rue, ait pu avoir une fonction commerciale ou artisanale. Les deux autres pièces constituent, en revanche, les parties utilitaires de l'habitation.

La pièce $\mathrm{Z}$ est un petit local qui paraît extérieur à la maison. Il ne donne cependant pas directement sur la rue, mais dans un espace assimilé par le fouilleur au porche d'entrée de la maison ouvert au sud sur la rue. La fouille partielle ${ }^{20} \mathrm{du}$ sol en terre battue, à la surface très cendreuse, a révélé de nombreux déchets métalliques

20. La surface du sol effectivement fouillée correspond à $35 \%$ de la pièce, soit environ $5 \mathrm{~m}^{2}$.

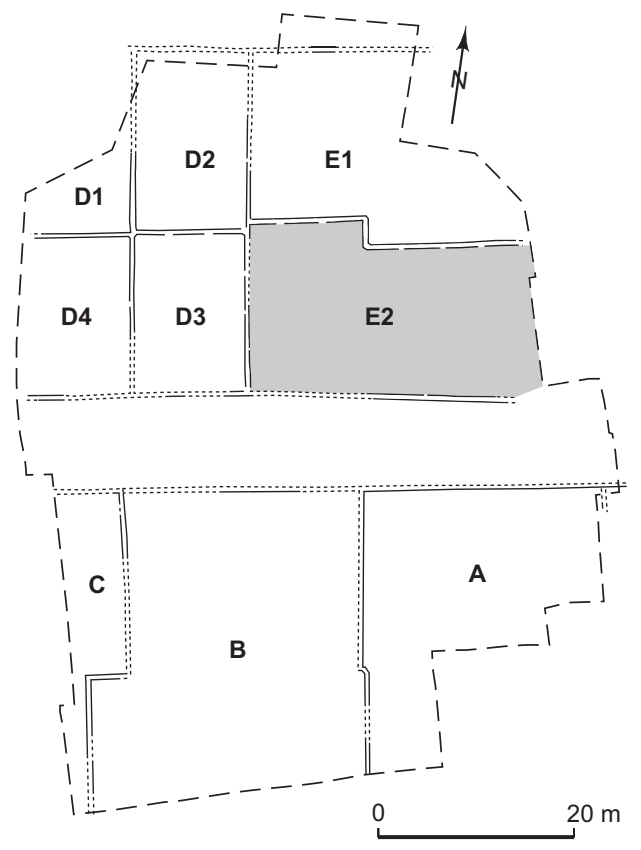

Fig. 2 - Localisation de la maison E2 dans le parcellaire de l'état II de Saint-Florent à Orange (DAO: P. Prévot, d'après données archéologiques : J.-M. Mignon, $S A D V)$.

suggérant la possible présence d'une activité liée au métal. De plus, la découverte d'éléments de placage en or, corail et bronze, ainsi que d'éléments de charnières en os pourrait suggérer la présence d'un espace dédié au montage d'éléments décoratifs sur des meubles ou coffrets. Si aucune structure artisanale n'a été formellement identifiée, le matériel retrouvé et la disposition de la pièce par rapport à l'ensemble de la domus pourraient suggérer son occupation par un atelier-boutique.

La cour principale (cour $\mathrm{n}^{\circ} 1$ ), d'une surface de $50 \mathrm{~m}^{2}$, occupe un espace de forme rectangulaire. Elle est en relation directe avec la résidence et revêt essentiellement un caractère ornemental. La cour secondaire (cour $\left.n^{\circ} 2\right)$, qui occupe elle aussi un espace rectangulaire et une superficie d'environ $50 \mathrm{~m}^{2}$, semble n'avoir qu'un caractère strictement utilitaire. C'est dans le puits de la cour no 2 (comblement 1531), situé au sud-est, que la série de réglettes en bois a été découverte (fig. 4). Elles étaient associées à des éléments de tablettes à écrire en bois ${ }^{21}$, de fuseaux en bois ${ }^{22}$ et à un étui cylindrique en bois ${ }^{23}$.

21. Éléments n ${ }^{\text {os }} 95.1 .547-551$ et 407 F-H.

22. Fuseaux nos 95.1.410-412 et 414.

23. Il s'agit du no 95.1.277. 


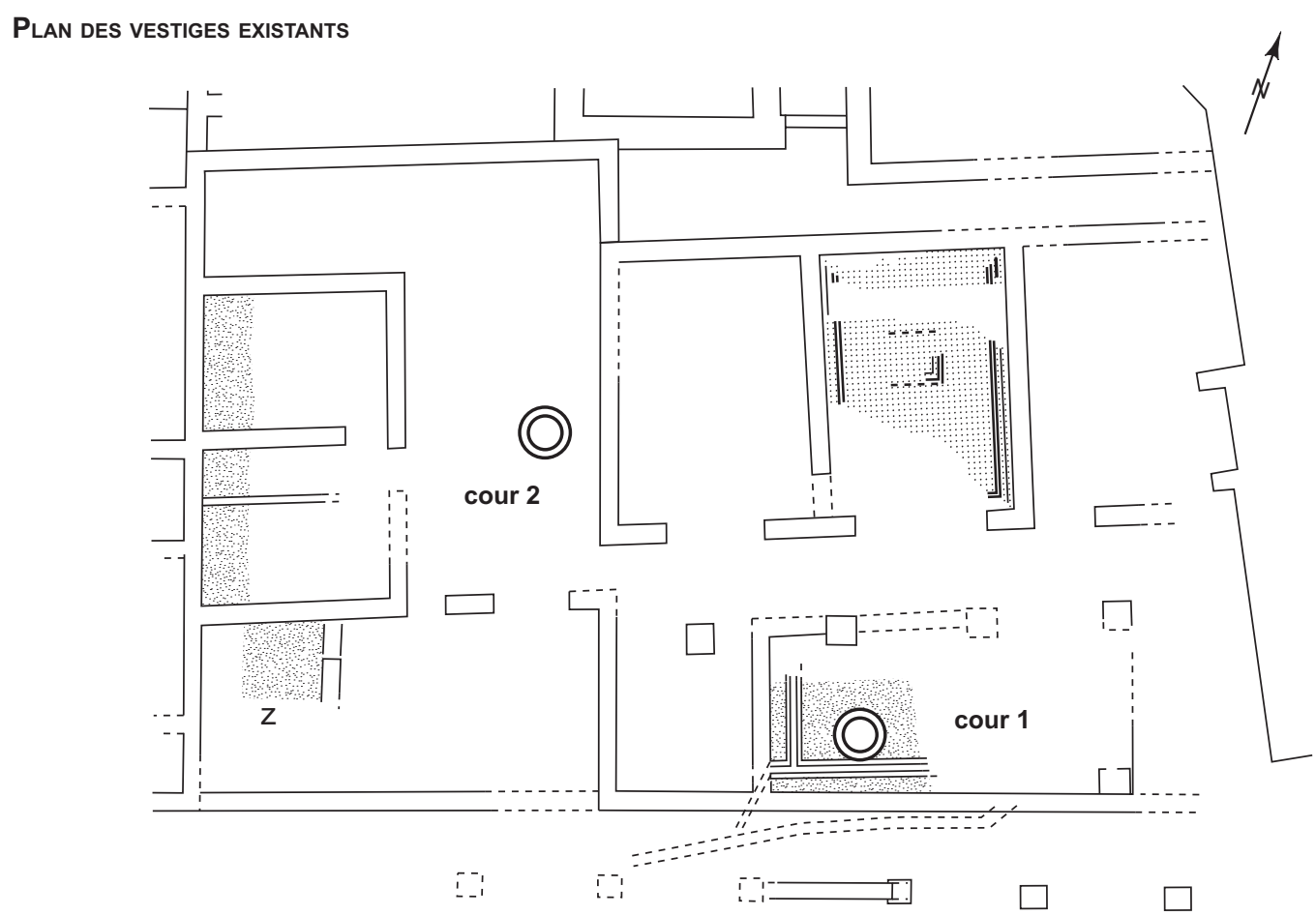

Plan restitué

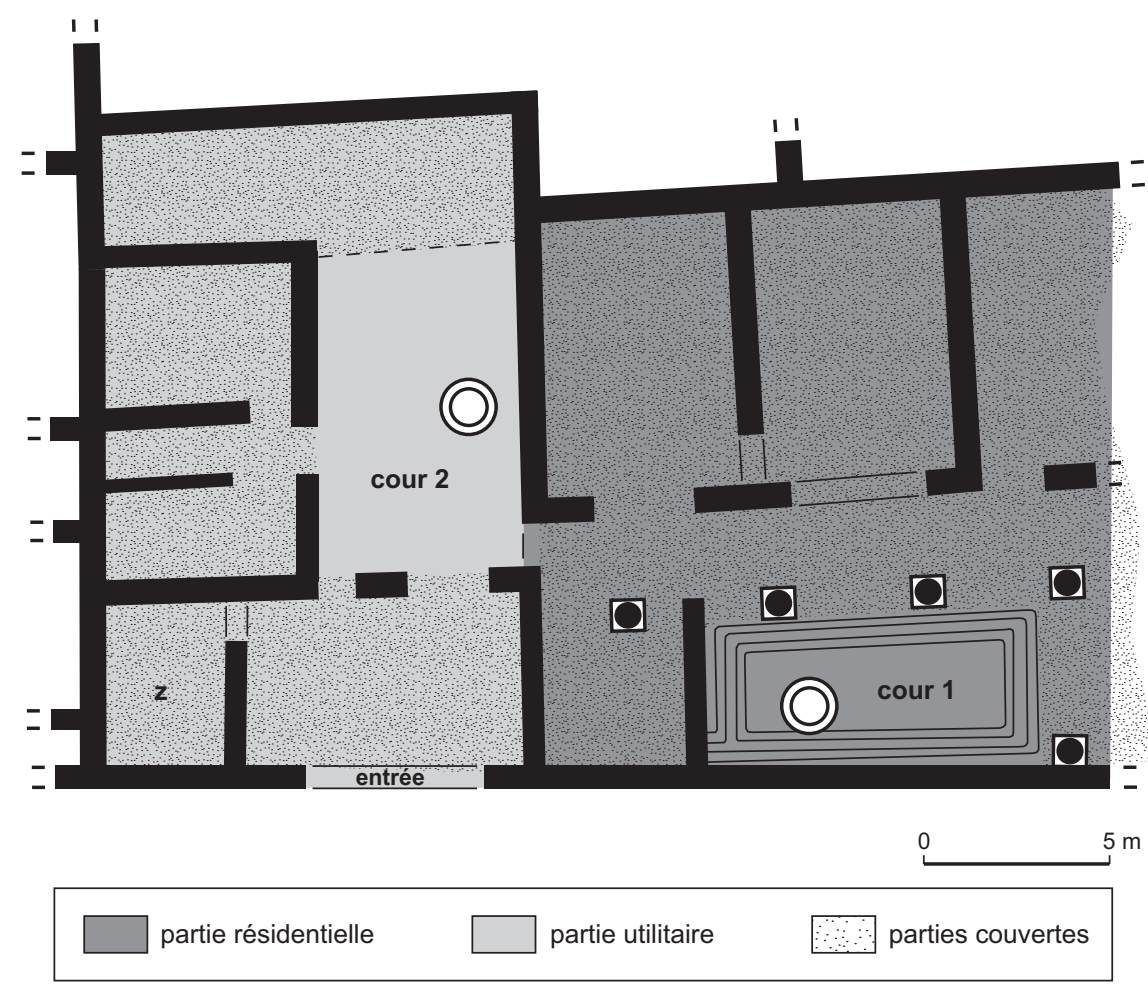

Fig. 3 - Plan de la maison E2 dans le parcellaire de l'état II de Saint-Florent à Orange. Les réglettes $n^{o s} 1-5$ proviennent du puits de la cour no 2 (DAO : P. Prévot, d'après Mignon, 1996, p. 275). 

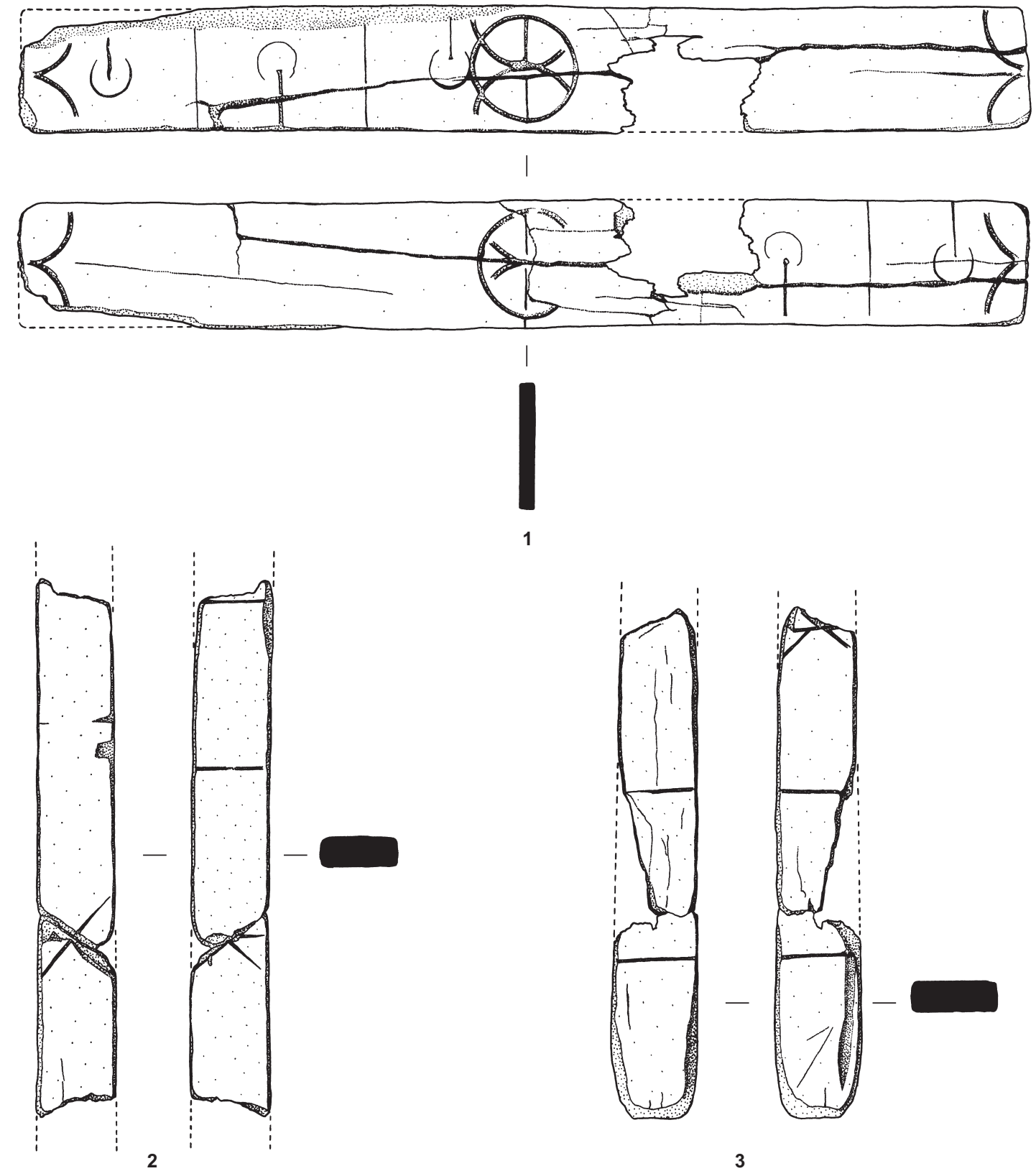

1
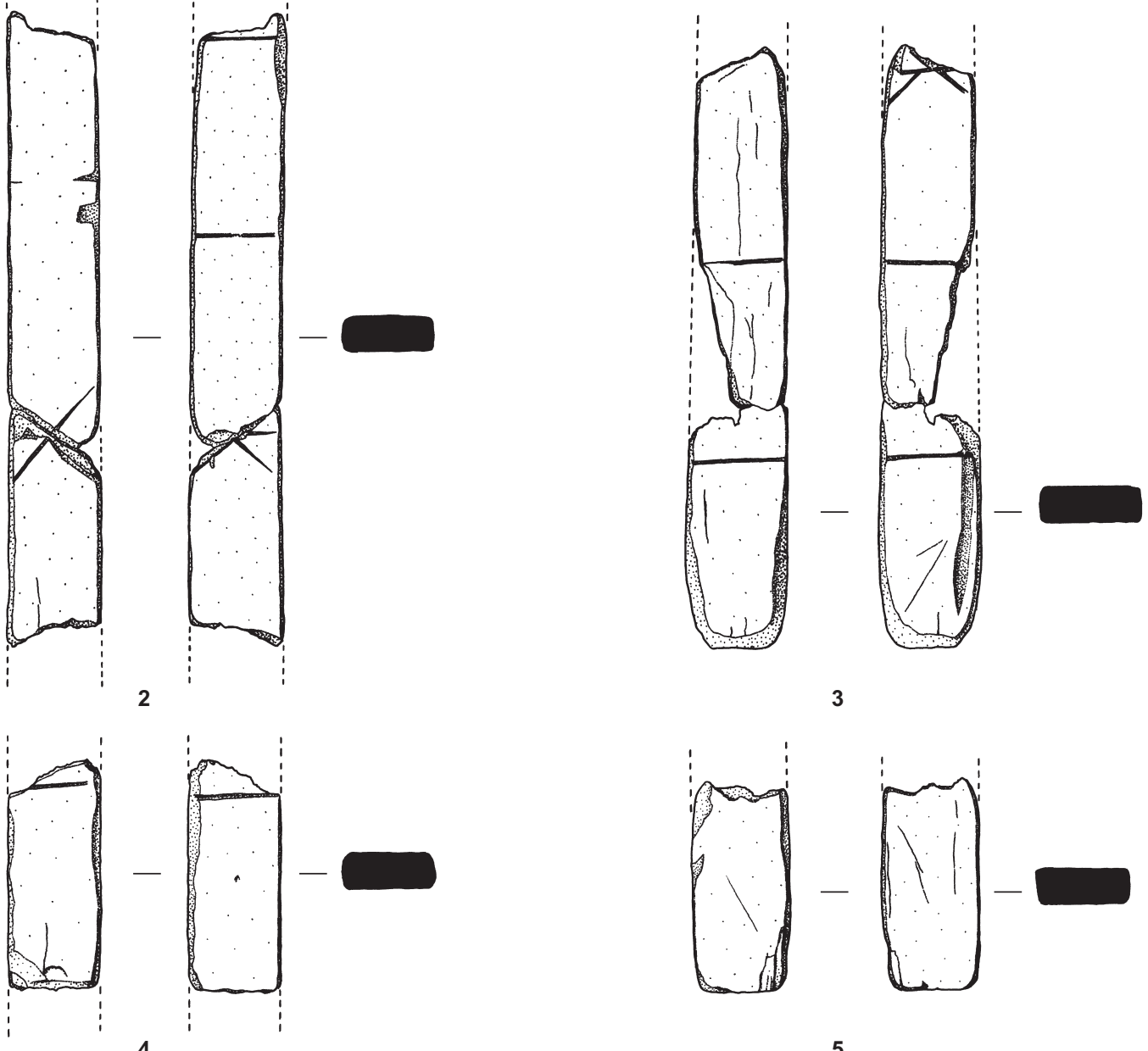

3

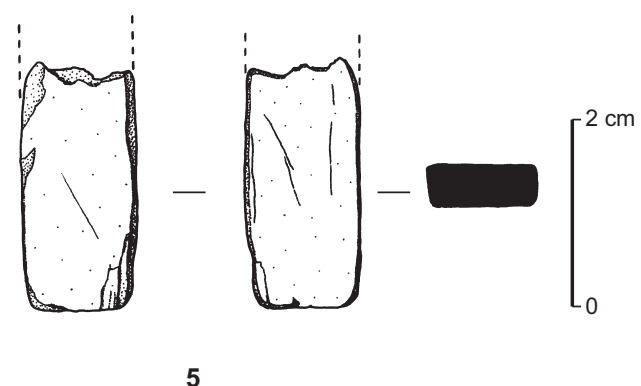

Fig. 4 - Série de réglettes en bois provenant de Saint-Florent à Orange. Elles sont étalonnées sur le demi-pied et ses subdivisions (dessins : P. Prévot). 
Nous avons choisi de diviser notre étude en deux parties distinctes. La première concerne la réglette complète $n^{\circ} 1$ $\left(n^{\circ} 95.1 .406\right)$ et la seconde, les réglettes $n^{\text {os }} 2$ à $5\left(n^{\circ} 95.1 .407\right.$ $\mathrm{A}, \mathrm{B} / \mathrm{C}$ et D-E). Cette différence de traitement tient aux caractéristiques morphologiques propres de chaque mesure, dont les dimensions et les types de graduations diffèrent.

\section{LES MESURES ORANGEOISES EN BOIS D'UN DEMI-PIED ROMAIN}

\section{LA RÉGLETTE COMPLÈTE No 1 ( No 95.1.406) $^{\circ}$}

Longue de $153 \mathrm{~mm}$ et large de $19 \mathrm{~mm}$, son épaisseur est comprise entre $2,20 \mathrm{~mm}$ et $2,40 \mathrm{~mm}$. Ses deux faces présentent des graduations transversales, réalisées alternativement des deux côtés, ainsi que des marques circulaires et semicirculaires. Ces graduations, vraisemblablement réalisées par incision, semblent s'apparenter aux subdivisions du pes monetalis (tabl. II). À savoir, sur chacune des deux faces : 1 demi-pied, 2 paumes, 3 pouces, 6 demi-pouces, 1 doigt et 4 demi-doigts (divisés en deux fois 2 demi-doigts).

Il s'agit, dans un premier temps, de s'interroger sur la façon dont la réglette a été étalonnée et de comparer les différentes subdivisions repérées à celles du pes monetalis théorique (tabl. II). Ainsi, nous avons mesuré, sur les faces A et $\mathrm{B}$ de la réglette, les dimensions de chaque subdivision du pied (fig. 5), et les avons transcrites dans le tableau III.

Le demi-pied, théoriquement égal à 147,80 mm, est compris entre les intersections des deux quarts de cercle tangents situés aux extrémités de la réglette. Il est égal, sur la face A, à 147,80 mm et, sur la face B, à 148,20 mm.

Chacune des 2 paumes (mesures A-B) est, quant à elle, comprise entre le point d'intersection des deux quarts de cercle tangents situés aux extrémités et le centre de la réglette. Celui-ci est marqué par un cercle dans lequel s'inscrivent deux demi-cercles tangents de même rayon et deux segments transversaux : le centre de la réglette est le point d'intersection des segments et des deux demi-cercles. La paume, théoriquement égale à $73,90 \mathrm{~mm}$, correspond, sur la face $\mathrm{A}$, à $74,40 \mathrm{~mm}$ pour la mesure A et $73,40 \mathrm{~mm}$ pour la mesure B. Sur la face B, la mesure A est égale $74,50 \mathrm{~mm}$ et la mesure B à $73,70 \mathrm{~mm}$.

L'une des deux paumes est ensuite divisée, grâce à deux segments transversaux de la largeur de la réglette, en trois pouces (mesures $\mathrm{C}-\mathrm{E}$ ). Le pouce, dont la valeur théorique équivaut à 24,60 mm, est égal, sur la face $\mathrm{A}$, à $24,10 \mathrm{~mm}$ pour la mesure $\mathrm{C}$, à $25,60 \mathrm{~mm}$ pour la mesure $\mathrm{D}$ et $23,70 \mathrm{~mm}$
Tabl. II - Mesures théoriques $d u$ semi-pes monetalis et de ses subdivisions: *, mesures relevées sur la réglette $n^{0} 1$.

\begin{tabular}{|c|c|c|c|c|c|c|c|}
\cline { 2 - 8 } \multicolumn{1}{c|}{} & Pes & Semi-pes & Palmus & Uncia & Semi-uncia & Digitus & Semi-digitus \\
\hline $\mathbf{1}$ & 295,7 & $147,8^{*}$ & $73,9^{*}$ & $24,6^{*}$ & $12,3^{*}$ & $18,5^{*}$ & $9,2^{*}$ \\
\hline $\mathbf{2}$ & & 295,7 & $147,8^{*}$ & $49,3^{*}$ & $24,6^{*}$ & 37 & $18,5^{*}$ \\
\hline $\mathbf{3}$ & & & & $73,9^{*}$ & $37^{*}$ & 55,4 & 27,7 \\
\hline $\mathbf{4}$ & & & & 98,6 & $49,3^{*}$ & 73,9 & 37 \\
\hline $\mathbf{5}$ & & & & 123,2 & $61,6^{*}$ & 92,4 & 46,2 \\
\hline $\mathbf{6}$ & & & & 147,8 & $73,9^{*}$ & 110,9 & 55,4 \\
\hline $\mathbf{7}$ & & & & & 86,2 & 129,4 & 64,7 \\
\hline $\mathbf{8}$ & & & & & 98,6 & 147,8 & 73,9 \\
\hline $\mathbf{9}$ & & & & & 110,9 & & 83,2 \\
\hline $\mathbf{1 0}$ & & & & & 123,2 & & 92,4 \\
\hline $\mathbf{1 1}$ & & & & & 135,5 & & 101,6 \\
\hline $\mathbf{1 2}$ & & & & & 147,8 & & 110,9 \\
\hline $\mathbf{1 3}$ & & & & & & & 120,1 \\
\hline $\mathbf{1 4}$ & & & & & & & 129,4 \\
\hline $\mathbf{1 5}$ & & & & & & & 138,6 \\
\hline $\mathbf{1 6}$ & & & & & & & 147,8 \\
\hline
\end{tabular}

1 pes $=2$ semi-pedes $=4$ palmi $=12$ unciae $=24$ semi-unciae $=16$ digit $=32$ semi-digit 1 semi-pes $=2$ palmi $=6$ unciae $=12$ semi-unciae $=8$ digiti $=16$ semi-digiti

1 palmus $=3$ unciae $=6$ semi-unciae $=4$ digit $i=8$ semi-digit $i$

1 uncia $=2$ semi-unciae

1 digitus $=2$ semi-digiti

pour la mesure $\mathrm{E}$; sur la face $\mathrm{B}$, il est égal à $23 \mathrm{~mm}$ pour la mesure C, 25,40 $\mathrm{mm}$ pour la mesure $\mathrm{D}$ et $26,10 \mathrm{~mm}$ pour la mesure E. De plus, chaque pouce comprend deux demipouces (mesures F-K) : ils sont marqués par des segments transversaux d'une longueur égale à la moitié de la largeur de la réglette. Chaque segment est surligné par les trois quarts d'un cercle. Le demi-pouce (théoriquement égal à $12,30 \mathrm{~mm}$ ) équivaut, en face $\mathrm{A}$, pour la mesure F à 11,40 $\mathrm{mm}$, pour la mesure $\mathrm{G}$ à $12,70 \mathrm{~mm}$, pour la mesure $\mathrm{H}$ à $13 \mathrm{~mm}$, pour la mesure I à $12,60 \mathrm{~mm}$, pour la mesure $\mathrm{J}$ à $13 \mathrm{~mm}$, enfin pour la mesure $\mathrm{K}$ à $10,70 \mathrm{~mm}$. En ce qui concerne la face $\mathrm{B}$, il est égal pour la mesure $\mathrm{F}$ à $10 \mathrm{~mm}$, pour les mesures $\mathrm{G}$ et $\mathrm{H}$ à $13 \mathrm{~mm}$, pour la mesure I à $12,40 \mathrm{~mm}$, pour la mesure J à 12,70 mm, enfin pour la mesure K à 13,40 mm.

Le doigt, quant à lui, équivaut à la largeur de la réglette. Théoriquement égal à $18,50 \mathrm{~mm}$, il mesure $19 \mathrm{~mm}$ sur les faces A et B.

Les points d'intersection des demi-cercles tangents, présents aux extrémités de la mesure et situés à la moitié de sa largeur, marquent les demi-doigts (mesures L-O). Ainsi, si pour les deux faces la mesure du doigt ne varie pas (puisque la largeur de la réglette est constante), celle des demi-doigts change en fonction de la place exacte du point d'intersection. Le demi-doigt, dont la valeur théorique correspond à $9,20 \mathrm{~mm}$, est égal, sur la face $\mathrm{A}$, à $8,80 \mathrm{~mm}$ pour 
Tabl. III - Mesures relevées sur la réglette $n^{0} 1$ et calcul de leur erreur par rapport aux subdivisions théoriques du pes monetalis.

\begin{tabular}{|c|c|c|c|c|}
\hline \multicolumn{2}{|c|}{ RÉGLETTE N $^{\circ} \mathbf{1}$} & Face A & Erreurs en mm & Erreurs en \% \\
\hline Semi-pes $(147,8 \mathrm{~mm})$ & 147,8 & 0 & 0 \\
\hline \multirow{2}{*}{$\begin{array}{c}\text { Palmus } \\
(73,9 \mathrm{~mm})\end{array}$} & $\mathrm{A}$ & 74,4 & 0,5 & 0,7 \\
\cline { 2 - 5 } & $\mathrm{B}$ & 73,4 & $-0,5$ & $-0,7$ \\
\hline \multirow{4}{*}{$\begin{array}{c}\text { Uncia } \\
(24,6 \mathrm{~mm})\end{array}$} & $\mathrm{C}$ & 24,1 & $-0,5$ & -2 \\
\cline { 2 - 5 } & $\mathrm{D}$ & 25,6 & 1 & 4,1 \\
\cline { 2 - 5 } & $\mathrm{E}$ & 23,7 & $-0,9$ & $-3,6$ \\
\hline \multirow{4}{*}{$\begin{array}{c}\text { Semi-uncia } \\
(12,3 \mathrm{~mm})\end{array}$} & $\mathrm{F}$ & 11,4 & $-0,9$ & $-7,3$ \\
\cline { 2 - 5 } & $\mathrm{G}$ & 12,7 & 0,4 & 3,2 \\
\cline { 2 - 5 } & $\mathrm{H}$ & 13 & 0,7 & 5,7 \\
\cline { 2 - 5 } & $\mathrm{J}$ & 13 & 0,1 & 0,8 \\
\cline { 2 - 5 } & $\mathrm{K}$ & 10,7 & $-1,6$ & -13 \\
\hline Digitus $(18,5 \mathrm{~mm})$ & 19 & 0,5 & 2,7 \\
\hline \multirow{4}{*}{$\begin{array}{c}\text { Semi-digitus } \\
(9,2 \mathrm{~mm})\end{array}$} & $\mathrm{L}$ & 8,8 & $-0,4$ & $-4,3$ \\
\cline { 2 - 5 } & $\mathrm{M}$ & 10,2 & 1 & 10,9 \\
\cline { 2 - 5 } & $\mathrm{N}$ & 8,9 & $-0,3$ & $-3,3$ \\
\cline { 2 - 5 } & $\mathrm{O}$ & 10,1 & 0,9 & 9,8 \\
\hline
\end{tabular}

\begin{tabular}{|c|c|c|}
\hline Face B & Erreurs en $\mathrm{mm}$ & Erreurs en \% \\
\hline 148,2 & 0,4 & 0,3 \\
\hline 74,5 & 0,6 & 0,8 \\
\hline 73,7 & $-0,2$ & $-0,3$ \\
\hline 23 & $-1,6$ & $-6,5$ \\
\hline 25,4 & 0,8 & 3,2 \\
\hline 26,1 & 1,5 & 6,1 \\
\hline 10 & $-2,3$ & $-18,7$ \\
\hline 13 & 0,7 & 5,7 \\
\hline 13 & 0,7 & 5,7 \\
\hline 12,4 & 0,1 & 0,8 \\
\hline 12,7 & 0,4 & 3,2 \\
\hline 13,4 & 1,1 & 8,9 \\
\hline 19 & 0,5 & 2,7 \\
\hline 9,8 & 0,6 & 6,5 \\
\hline 9,2 & 0 & 0 \\
\hline 9,5 & 0,3 & 3,3 \\
\hline \multirow[t]{3}{*}{9,5} & 0,3 & 3,3 \\
\hline & 0,7 & 4,5 \\
\hline & 0,37 & \\
\hline
\end{tabular}

la mesure $\mathrm{L}$, à 10,20 $\mathrm{mm}$ pour la mesure $\mathrm{M}$, à $8,90 \mathrm{~mm}$ pour la mesure $\mathrm{N}$ et à $10,10 \mathrm{~mm}$ pour la mesure $\mathrm{O}$. Concernant la face $\mathrm{B}$, les valeurs du demi-doigt sont égales à $9,80 \mathrm{~mm}$ pour la mesure $\mathrm{L}$, à 9,20 $\mathrm{mm}$ pour la mesure $\mathrm{M}$ et à 9,50 $\mathrm{mm}$ pour les mesures $\mathrm{N}$ et $\mathrm{O}$.

Nous pouvons, à présent, comparer les dimensions mesurées sur la réglette $\mathrm{n}^{\mathrm{o}} 1$ à celles des subdivisions du pes monetalis. Il s'agit alors de faire la différence entre la mesure relevée et la mesure théorique (tabl. III), celle-ci pouvant être interprétée comme un coefficient d'erreur: nous l'avons exprimée en millimètres, puis en pourcentage ${ }^{24}$.

Le demi-pied, sur la face A, est égal à sa valeur théorique. L'erreur est donc nulle. En revanche, sur la face B, il mesure $0,40 \mathrm{~mm}(0,3 \%)$ de plus que sa valeur théorique.

Les valeurs de la paume, sur la face $A$, diffèrent de $0,50 \mathrm{~mm}(0,7 \%)$ en A et de - 0,50 mm (-0,7 \%) en B. Sur la face $\mathrm{B}$, la différence est égale, pour la mesure $\mathrm{A}$, à $0,60 \mathrm{~mm}$ $(0,8 \%)$ et à $0,20 \mathrm{~mm}(0,3 \%)$ pour la mesure B.

Les valeurs du coefficient d'erreur du pouce, sur la face $\mathrm{A}$, sont égales à - 0,50 mm (- $2 \%)$ pour la mesure $\mathrm{C}$, à $10 \mathrm{~mm}(4,1 \%)$ pour la mesure D et à $-0,90 \mathrm{~mm}(-3,6 \%)$ pour la mesure E. Sur la face B, elles sont égales à - 1,60 mm

24. Pour obtenir le pourcentage d'erreur, nous avons multiplié par 100 le nombre erroné, et divisé le total obtenu par la valeur théorique.
$(-6,5 \%)$ pour la mesure $\mathrm{C}$, à $0,80 \mathrm{~mm}(3,2 \%)$ pour la mesure $\mathrm{D}$ et à $1,50 \mathrm{~mm}(6,1 \%)$ pour la mesure $\mathrm{E}$.

Le demi-pouce, quant à lui, présente une erreur, en face $\mathrm{A}$, de $-0,90 \mathrm{~mm}(-7,3 \%)$ pour la mesure $\mathrm{F}$, de $0,40 \mathrm{~mm}(3,2 \%)$ pour la mesure $\mathrm{G}$, de $0,70 \mathrm{~mm}(5,7 \%)$ pour la mesure $\mathrm{H}$, de $0,10 \mathrm{~mm}(0,8 \%)$ pour la mesure $\mathrm{I}$, de $0,70 \mathrm{~mm}(5,7 \%)$ pour la mesure $\mathrm{J}$ et de $-1,60 \mathrm{~mm}$ $(-13 \%)$ pour la mesure K. Sur la face B, elle est égale à $-2,30 \mathrm{~mm}(-18,7 \%)$ pour la mesure F, à $0,70 \mathrm{~mm}(5,7 \%)$ pour les mesures $\mathrm{G}$ et $\mathrm{H}$, à $0,10 \mathrm{~mm}(0,8 \%)$ pour la mesure I, à $0,40 \mathrm{~mm}(3,2 \%)$ pour la mesure J et à $1,10 \mathrm{~mm}(8,9 \%)$ pour la mesure $\mathrm{K}$.

Le coefficient d'erreur du doigt, pour les faces A et B, est de $0,50 \mathrm{~mm}(2,7 \%)$.

Enfin, le demi-doigt, présente une erreur, en face A, de - 0,40 mm (-4,3\%) pour la mesure L, de $1 \mathrm{~mm}(10,9 \%)$ pour la mesure $\mathrm{M}$, de $-0,30 \mathrm{~mm}(-3,3 \%)$ pour la mesure $\mathrm{N}$ et de $0,90 \mathrm{~mm}(9,8 \%)$ pour la mesure O. En face B, elle est égale à $0,60 \mathrm{~mm}(6,5 \%)$ pour la mesure L. La mesure $\mathrm{M}$ correspond, en revanche, à la valeur du demi-doigt théorique, c'est-à-dire 9,20 mm. Les mesures $\mathrm{N}$ et $\mathrm{O}$ présentent, quant à elles, une erreur de 0,30 mm (3,3\%).

Nous pouvons calculer, alors, le degré de précision globale de la réglette $\mathrm{n}^{\circ} 1$. Il s'agit, premièrement, de calculer 
FAce A

$1 / 2$ pes
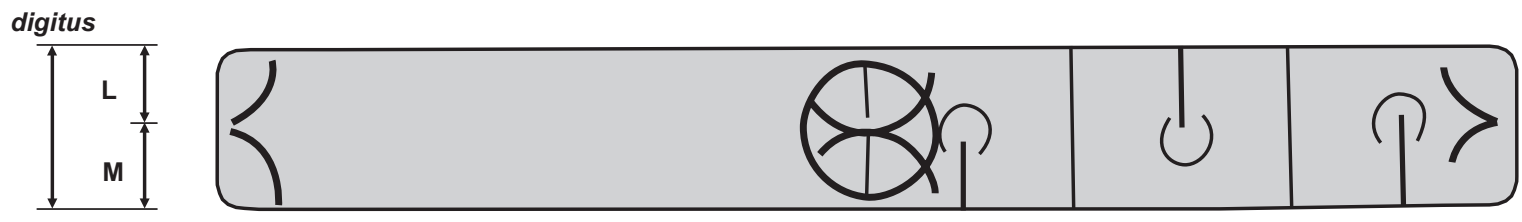

$1 / 2$ digitus

B

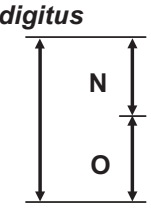

$1 / 2$ digitus

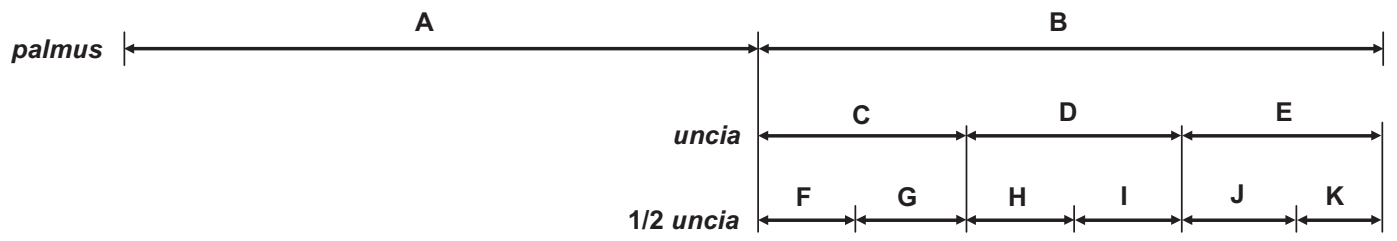

FACE B

$1 / 2$ pes
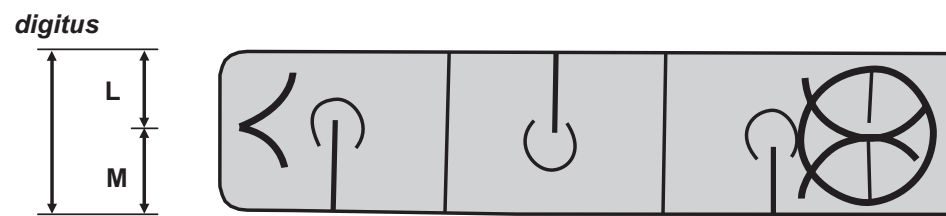

$1 / 2$ digitus

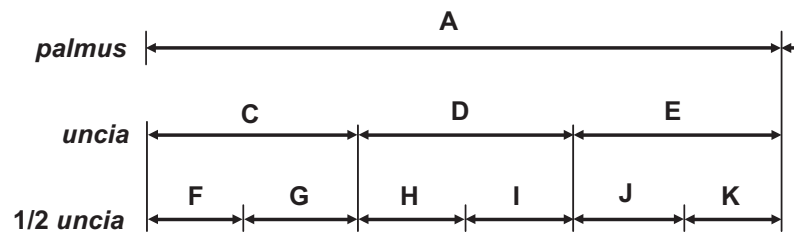

Fig. 5 - Schéma de lecture des graduations de la réglette $n^{\circ} 1$ (DAO : P. Prévot).

la moyenne des erreurs pour chacune des faces. Elle est de $0,60 \mathrm{~mm}(4,6 \%)$ pour la face A et de $0,70 \mathrm{~mm}(4,5 \%)$ pour la face B (tabl. III). Deuxièmement, et à partir des valeurs que nous venons de calculer, nous allons déterminer la moyenne des erreurs pour l'ensemble des valeurs des faces A et $\mathrm{B}$. Elle s'élève à $0,70 \mathrm{~mm}(5 \%)$. La réglette $\mathrm{n}^{\mathrm{o}} 1$, étalonnée sur le demi-pied et ses subdivisions, est précise à $95 \%$.

Nous pouvons poser ensuite la question du rapport entre les faces A et B de la réglette $n^{\circ} 1$. En effet, les erreurs mesurées (en millimètres) pour les graduations de chacune des faces présentent-elles des similitudes significatives? Afin de répondre à cette question, il faut effectuer un test sur l'égalité des moyennes entre ces deux lots.

Il convient de vérifier au préalable, et grâce au test du F de Fischer ${ }^{25}$ (Chenorkian, 1996, p. 24-27), si les variantes des deux échantillons diffèrent significativement ou non (tabl. IV). Comme $\mathrm{n} 1=\mathrm{n} 2$ et que $\mathrm{S}^{2}<\mathrm{S} 2^{2}, \mathrm{~F}=\mathrm{S} 2^{2} / \mathrm{S}^{2}$, nous constatons que $\mathrm{F}=0,41$. En se reportant aux tables des

25. La vérification de l'absence de variance de deux échantillons se calcule de la manière suivante $: \mathrm{F}=\mathrm{S}^{2}{ }^{2} / \mathrm{S}^{2}$. 
Tabl. IV - Résultats du calcul du F de Fischer, par face de la réglette $n^{0} 1$, appliqué aux moyennes totales des erreurs des mesures relevées par rapport aux subdivisions théoriques.

\begin{tabular}{|l|c|c|}
\hline \multicolumn{1}{|c|}{ RÉGLETTE N $^{\circ} \mathbf{1}$} & Face A & Face B \\
\hline Moyenne (M) & 0,64 & 0,71 \\
\hline Variance (S $\leq$ ) & 0,15 & 0,36 \\
\hline Observations (N) & 17 & 17 \\
\hline Degré de liberté (Ddl) & 16 & 16 \\
\hline F & \multicolumn{2}{|c|}{$\mathbf{0 , 4 1}$} \\
\hline P(F<=f) unilatéral & \multicolumn{2}{|c|}{0,04} \\
\hline Valeur critique pour F (unilatéral) & \multicolumn{2}{|c|}{0,42} \\
\hline
\end{tabular}

Tabl. VI - Résultats du calcul de la moyenne des erreurs par types de subdivisions sur la réglette $n^{0} 1$.

\begin{tabular}{|l|c|c|c|}
\hline RÉGLETTE N $^{\mathbf{1}}$ & Face A & Face B & Total \\
\hline Semi-pes & $0 \%$ & $0,3 \%$ & $\mathbf{0 , 2} \%$ \\
\hline Palmus & $0,7 \%$ & $0,6 \%$ & $\mathbf{0 , 6} \%$ \\
\hline Uncia & $3,2 \%$ & $5,3 \%$ & $\mathbf{4 , 3} \%$ \\
\hline Semi-uncia & $6 \%$ & $7,2 \%$ & $\mathbf{6 , 6} \%$ \\
\hline Digitus & $2,7 \%$ & $2,7 \%$ & $\mathbf{2 , 7} \%$ \\
\hline Semi-digitus & $7,1 \%$ & $3,3 \%$ & $\mathbf{5 , 2} \%$ \\
\hline
\end{tabular}

valeurs de F (Chenorkian, 1996, p. 142-147, tables 1-3), nous remarquons que la valeur critique est de 0,42 (au seuil de $0,05)$; puisque $0,41<0,42$, alors $0,05<\mathrm{P}(\mathrm{F})$. La différence entre les variantes des deux échantillons n'est donc pas significative.

Nous pouvons ensuite procéder au test paramétrique de l'égalité des moyennes entre ces deux lots, grâce au test du T de Student ${ }^{26}$ (tabl. V) (Chenorkian, 1996, p. 30-35). Nous constatons que $\mathrm{T}=0,4$. En se reportant à la table de distribution de T (Chenorkian, 1996, p. 149, table 5), nous remarquons que la valeur critique est de 2,4 (au seuil de $0,05)$. En valeur absolue, T est inférieur à la valeur critique : il n'y a donc pas de différence significative entre les deux moyennes; nous pouvons même dire que cette absence de différence est hautement significative, puisque $\mathrm{T}$ est inférieur à la valeur critique (au seuil de 0,001).

$\mathrm{Au}$ final, il semble que les moyennes des erreurs (en millimètres) de la totalité des mesures, relevées sur chacune des faces A et B, présentent des similitudes significatives.

26. Il s'agit, dans un premier temps, de calculer la variante $\left(\mathrm{S}^{2}\right)$ de l'échantillon à partir des effectifs (N1 et N2), de la somme des valeurs (x1 et $\mathrm{x} 2$ ) de la moyenne des valeurs (xi) de chacune des séries A et $\mathrm{B}$ : $\mathrm{S}^{2}=\left(\Sigma[\mathrm{xi}-\mathrm{x} 1]^{2}+\Sigma[\mathrm{xi}-\mathrm{x} 2]^{2}\right) /(\mathrm{N} 1+\mathrm{N} 2)-2$. Le T de Student peut, dans un second temps, être calculé $: \mathrm{T}=(\mathrm{m} 1-\mathrm{m} 2) / \sqrt{\left(\left[\mathrm{S}^{2} / \mathrm{N} 1\right]+\left[\mathrm{S}^{2} / \mathrm{N} 2\right]\right)}$.
Tabl. V - Résultats du calcul du T de Student, par face de la réglette $n^{o} 1$, appliqué aux moyennes totales des erreurs des mesures relevées par rapport aux subdivisions théoriques.

\begin{tabular}{|c|c|c|}
\hline RÉGLetTe N 1 & Face A & Face B \\
\hline Moyenne (M) & 0,71 & 0,64 \\
\hline Variance $(\mathrm{S} \leq$ ) & 0,36 & 0,15 \\
\hline Observations (N) & 17 & 17 \\
\hline Variance pondérée & \multicolumn{2}{|c|}{0,26} \\
\hline Différence hypothétique des moyennes & \multicolumn{2}{|c|}{0} \\
\hline Degré de liberté (Ddl) & \multicolumn{2}{|c|}{32} \\
\hline Statistique T & \multicolumn{2}{|c|}{0,41} \\
\hline $\mathrm{P}(\mathrm{T}<=\mathrm{t})$ unilatéral & \multicolumn{2}{|c|}{0,34} \\
\hline Valeur critique pour t (unilatéral) & \multicolumn{2}{|c|}{1,69} \\
\hline $\mathrm{P}(\mathrm{T}<=\mathrm{t})$ bilatéral & \multicolumn{2}{|c|}{0,69} \\
\hline Valeur critique pour $\mathrm{t}$ (bilatéral) & \multicolumn{2}{|c|}{2,04} \\
\hline
\end{tabular}

Concernant les processus techniques de fabrication de cette réglette, nous pouvons nous interroger sur la manière dont les subdivisions et les graduations ont été réalisées. Il convient, dans un premier temps, de s'attarder sur le pourcentage total des erreurs, par type de subdivision (tabl. VI).

Le pourcentage total d'erreurs du demi-pied, relevé sur les faces A et B, est de $0,2 \%, 0,6 \%$ pour la paume, 4,3\% pour le pouce, $6,6 \%$ pour le demi-pouce, $2,7 \%$ pour le doigt et $5,2 \%$ pour le demi-doigt.

Nous constatons, comme le montre la figure 6, que le pourcentage d'erreurs évolue en fonction de deux critères. D'une part, selon l'ensemble auquel appartient la subdivision, à savoir l'ensemble des mesures réparties soit sur la longueur de la réglette, soit sur sa largeur. D’autre part, selon les valeurs des types de subdivisions. En effet, nous observons que plus le type de mesure est petit, plus son pourcentage d'erreurs augmente. Par exemple, sur la longueur de la réglette le demi-pied a été globalement réalisé avec plus de précision que le demi-pouce.

Il semble que cette observation repose directement sur la façon dont a été fabriquée et étalonnée la réglette $\mathrm{n}^{\circ} 1$. Ainsi, nous pouvons proposer une hypothèse de fabrication de la mesure : nous distinguons sept étapes théoriques de fabrication et d'étalonnage ${ }^{27}$ (fig. 7). Selon nous, l'artisan est parti d'une plaquette de bois dont l'épaisseur a été préalablement débitée.

27. Il s'agit d'une interprétation liée à l'analyse des mesures et des graduations dans laquelle nous ne distinguons pas les faces A ou B. 


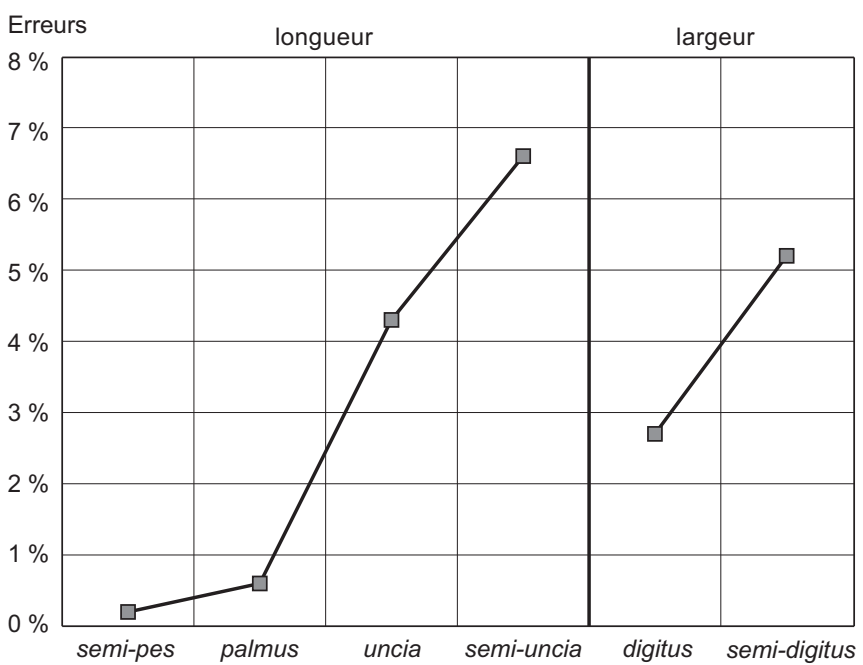

Fig. 6 - Moyenne totale des erreurs par types de mesures sur la réglette $n^{\circ} 1$ (DAO:P. Prévot).

- Durant la première étape, il a procédé à la délimitation, probablement par incision, de la largeur de la future réglette et à son débitage longitudinal. Il est vraisemblable qu'il se soit servi d'un étalon du doigt. Ainsi, la première étape consiste en l'étalonnage de la largeur de la future réglette sur le doigt et s'achève par un débitage.

- La deuxième étape, quant à elle, consiste en l'étalonnage du demi-pied. L'artisan, probablement au moyen d'un étalon, a réalisé des trous de pointage délimitant la longueur du demi-pied. Ceux-ci se situent à la moitié de la largeur. Il a ensuite débité transversalement la plaquette et arrondi les angles, lui donnant ainsi sa forme définitive.

- La troisième étape correspond au ciselage des graduations périphériques qui marquent le demi-pied et les demi-doigts. Celles-ci se présentent sous la forme de deux quarts de cercles tangents, peut-être tracés au compas, dont l'intersection coïncide avec la graduation.

- La quatrième étape consiste en la séparation en deux moitiés égales de la mesure du demi-pied, de façon à obtenir deux paumes. Le fait que chacune des moitiés ne présente pas la même longueur signifie que l'artisan ne s'est pas servi d'un étalon de la paume pour les tracer, il a réalisé un trou de pointage à l'œil.

- La cinquième étape s'apparente alors au ciselage des graduations qui séparent le demi-pied en deux moitiés. L'artisan a tracé un cercle à partir du trou de pointage, puis a ensuite réalisé, à l'intérieur du premier cercle, deux demi-cercles tangents dont le point d'intersection est le trou de pointage. Il est possible que ceux-ci aient été tracés au compas. Il est intéressant de constater que cette marque centrale, probablement ciselée de cette manière pour qu'on la repère d'un coup d'œil, est comparable à celles gravées sur les réglettes en bois d'if de Vindonissa, Suisse (Fellmann, 1988, p. 200, no 159 ).

- La sixième étape consiste au traçage des graduations transversales qui marquent le pouce. L'artisan a réalisé trois segments transversaux : il semble qu'il ait divisé la paume en trois tiers approximatifs; en effet, aucune des mesures du pouce ne présente les mêmes dimensions, cela signifie qu'il n'a pas utilisé d'étalon du pouce pour les réaliser, mais qu'il les a tracées à l'œil.

C'est aussi le cas des demi-segments transversaux qui séparent les pouces en deux. Ils ont, selon toute vraisemblance, été tracés de façon approximative, puis ont été soulignés de quarts de cercles, peut-être destinés à repérer la graduation d'un coup d'œil.

L'étalonnage du demi-pied et du doigt semble avoir été réalisé à l'aide d'un étalon métallique ou au moyen d'une règle. Cette hypothèse explique, selon nous, le caractère « exponentiel» de la répartition des pourcentages par type de mesure. En effet, si les mesures du demi-pied et du doigt sont quasi exactes, ce n'est pas le cas de leurs subdivisions respectives qui semblent avoir été tracées à l'œil.

Il semble, au final, que la réglette $\mathrm{n}^{\circ} 1$ ait été bel et bien étalonnée sur le semi-pes monetalis et ses subdivisions. Bien que les mesures des subdivisions comportent des erreurs, dues selon nous au facteur humain, cette réglette reste extrêmement précise puisque l'erreur ne dépasse quasiment jamais le $1 / 10^{\mathrm{e}}$ de millimètre. Elles restent très proches des subdivisions du pes monetalis théorique (tabl. II). Enfin, il convient de souligner la maniabilité de cet instrument. En effet, les subdivisions de la paume, graduées alternativement d'un côté ou de l'autre, permettent une lecture aisée des mesures quelle que soit la prise en main de la réglette. $\mathrm{Si}$ l'artisan se trompait de côté, il lui suffisait simplement de la retourner. Les graduations, faciles à lire, de cette mesure en bois étaient probablement destinées à l'artisanat de petits objets nécessitant l'usage du demi-doigt.

\section{LES FRAGMENTS DES RÉGLETTES Nos 2 À 5 (No 95.1.407 A, B/C ET D-E)}

Les réglettes $\mathrm{n}^{\text {os }} 2$ à 5 sont pourvues de caractères morphologiques communs. D'une part, elles sont en buis (Buxus sempervirens) et, d'autre part, elles sont graduées de façon similaire, étalonnées sur le semi-pes, l'uncia et la semi-uncia. Enfin, leur largeur varie de $12,10 \mathrm{~mm}$ à $12,60 \mathrm{~mm}$ et leur épaisseur de $3,50 \mathrm{~mm}$ à $4,70 \mathrm{~mm}$. 

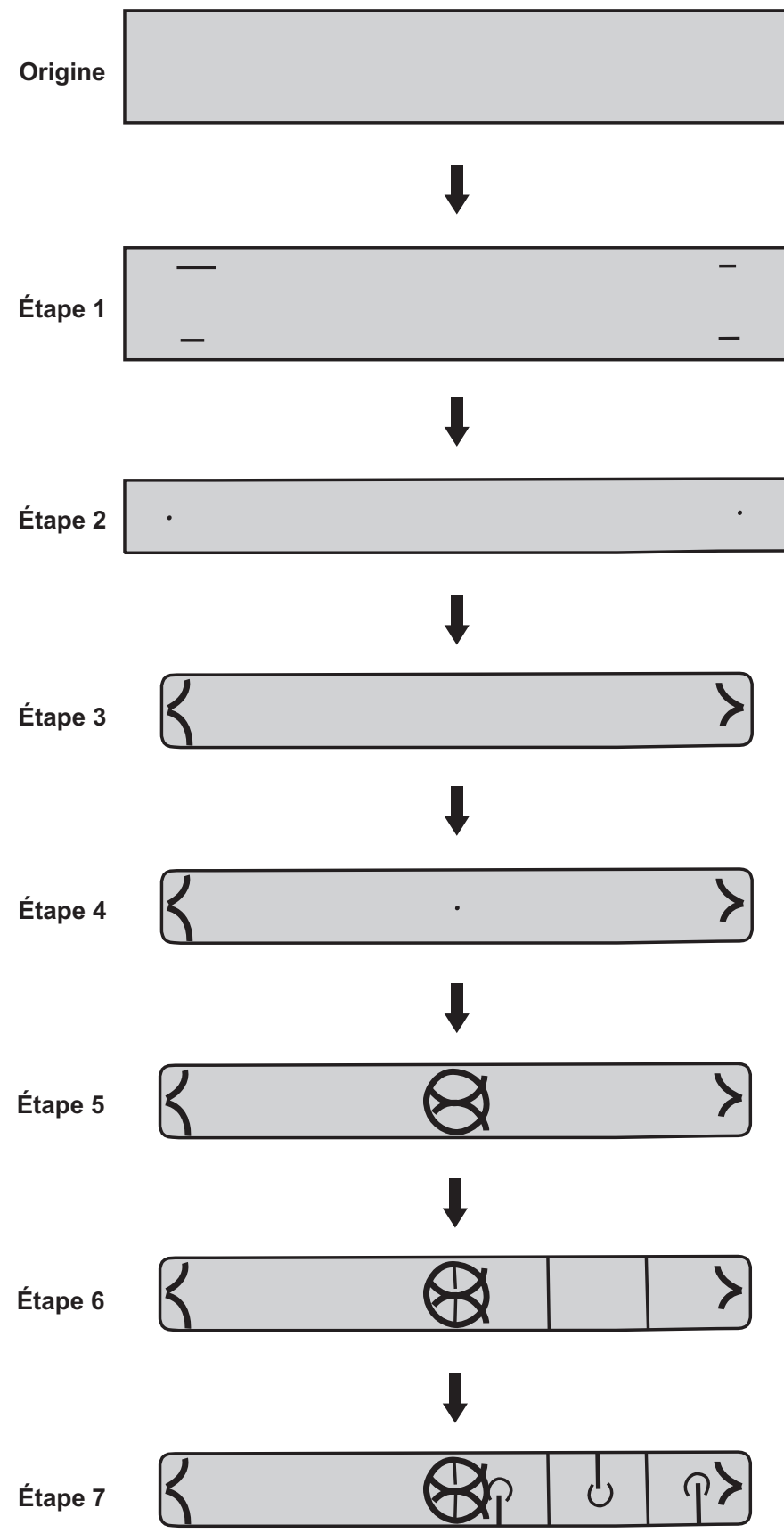

Plaquette de bois

Délimitation de la largueur (égale au digitus), suivie d'un débitage longitudinal

Étalonnage du semi-pes par des trous de pointage, suivi d'un débitage transversal

Ciselage des graduations périphériques qui marquent le semi-pes et les semi-digiti Étalonnage du palmus
par un trou de pointage

Ciselage des graduations centrales qui marquent le palmus

Ciselage des graduations transversales qui marquent l'uncia

Ciselage des graduations transversales et circulaires qui marquent les semi-unciae

Fig. 7 - Reconstitution des étapes techniques de fabrication de la réglette $n^{o} 1$ (DAO : P. Prévot).

Il s'agit, dans un premier temps, de mesurer chaque graduation, puis de les comparer aux subdivisions théoriques du pes monetalis en procédant à l'analyse des erreurs réalisées par l'artisan. Ainsi, nous pourrons donner une échelle de précision pour chacune des réglettes. Dans un deuxième temps, nous tenterons d'étudier la répartition des graduations sur les faces des réglettes, de manière à comprendre comment elles étaient utilisées.

Le nombre réduit des données relevées, au contraire de celles de la réglette $\mathrm{n}^{\circ} 1$, ne permettra pas de leur appliquer des tests paramétriques. 
Tabl. VII - Mesures relevées sur la réglette no 2 et calcul de leur erreur par rapport aux subdivisions théoriques du pes monetalis.

\begin{tabular}{|c|c|c|c|c|}
\hline \multicolumn{2}{|c|}{ RÉGLETTE N ${ }^{\circ} \mathbf{2}$} & Face A & Erreurs en mm & Erreurs en \% \\
\hline \multirow{2}{*}{$\begin{array}{c}\text { Uncia } \\
(24,6 \mathrm{~mm})\end{array}$} & $\mathrm{A}$ & - & - & - \\
\cline { 2 - 5 } & $\mathrm{B}$ & - & - & - \\
\cline { 2 - 5 } & $\mathrm{C}$ & - & - & - \\
\hline $\begin{array}{c}\text { Semi-uncia } \\
(12,3 \mathrm{~mm})\end{array}$ & $\mathrm{D}$ & 12,1 & $-0,2$ & $-1,6$ \\
\hline
\end{tabular}

\begin{tabular}{|c|c|c|}
\hline Face B & Erreurs en mm & Erreurs en \% \\
\hline 24,5 & $-0,1$ & $-0,4$ \\
\hline 25 & 0,4 & 1,6 \\
\hline 25 & 0,4 & 1,6 \\
\hline 12,1 & $-0,2$ & $-1,6$ \\
\hline & 0,3 & 1,3 \\
\hline
\end{tabular}

FACE A

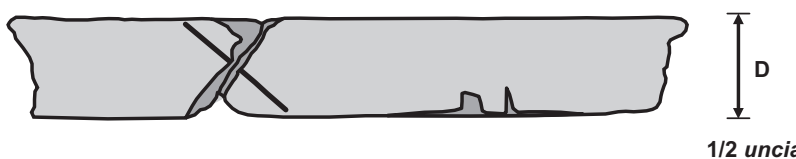

FACE B

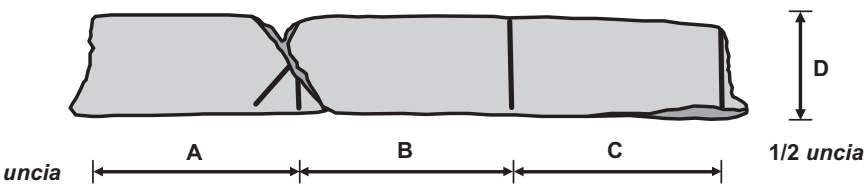

Fig. 8 - Schéma de lecture des graduations de la réglette $n^{0} 2$ (DAO : P. Prévot).

\section{ANALYSE MÉTROLOGIQUE DU LOT DE RÉGLETTES N $\mathrm{N}^{\mathrm{OS}} 2$ À 5}

\section{La réglette $n^{\circ} 2\left(n^{\circ}\right.$ 95.1.407 A)}

La réglette $\mathrm{n}^{\circ} 2$ est longue de $81 \mathrm{~mm}$, sa largeur est de $12,10 \mathrm{~mm}$ et son épaisseur varie entre $4,60 \mathrm{~mm}$ et 4,70 $\mathrm{mm}$. Les deux extrémités de la réglette ont été brisées. Sa face A possède une incision diagonale, tandis que sa face $\mathrm{B}$ porte une incision diagonale et trois incisions verticales.

Nous avons mesuré, sur les faces A et B, les dimensions comprises entre chaque graduation (fig. 8 et tabl. VII).

Le pouce (théoriquement égal à 24,60 mm) est compris entre les segments verticaux de la face B (mesures A-C). La mesure A est égale à 24,50 mm, la mesure B à $25 \mathrm{~mm}$ et la mesure C à $25 \mathrm{~mm}$.

Le demi-pouce (théoriquement égal à 12,30 mm) est égal à la largeur de la réglette (mesure D). Il mesure 12,10 mm sur les faces A et B.

Le fait que la réglette soit brisée à ses deux extrémités est à prendre en compte. En effet, nous ne pouvons mesurer que les subdivisions visibles et il semble qu'elle ait aussi été étalonnée sur des valeurs supérieures au pouce : peut-être le demi-pied ou la paume. Ainsi, nous pouvons comparer les dimensions des graduations mesurées sur la réglette aux valeurs théoriques du pouce et du demi-pouce, de manière à calculer le coefficient d'erreur (tabl. VII).

Le pouce, sur la face $B$, présente une erreur de $-0,10 \mathrm{~mm}(-0,4 \%)$ pour la mesure A et de $0,40 \mathrm{~mm}$ $(1,6 \%)$ pour les mesures $\mathrm{B}$ et $\mathrm{C}$.

Le demi-pouce, quant à lui, est erroné de $-0,20 \mathrm{~mm}$ $(-1,6 \%)$ pour la mesure $\mathrm{D}$ et sur chacune des faces de la réglette.

Il s'agit, à présent, de déterminer le degré global de précision de la réglette $\mathrm{n}^{\circ} 2$ en calculant la moyenne des erreurs pour chaque face. Elle est de $0,20 \mathrm{~mm}(1,6 \%)$ pour la face A et de $0,30 \mathrm{~mm}(1,3 \%)$ pour la face B (tabl. VII). Nous pouvons alors déterminer la moyenne des erreurs pour l'ensemble des valeurs des faces A et B : elle est égale à $0,20 \mathrm{~mm}(1,5 \%)$. Les mesures relevées sur la réglette $\mathrm{n}^{\circ} 2$, étalonnées sur le pouce et le demi-pouce, sont donc précises à $98,5 \%$.

\section{La réglette $n^{\circ} 3\left(n^{\circ} 95.1 .407 \mathrm{~B} / \mathrm{C}\right)$}

La réglette $\mathrm{n}^{\circ} 3$ est longue de 77,10 mm, sa largeur est de 12,60 mm et son épaisseur de 4,40 mm. L'une des deux extrémités a été brisée. Sa face A possède deux incisions verticales. Sa face B, quant à elle, comprend trois incisions verticales : l'une d'elle est superposée à deux incisions diagonales qui forment un $\mathrm{X}$.

Nous avons mesuré, sur les faces A et B, les dimensions comprises entre chaque graduation (fig. 9 et tabl. VIII).

La paume (théoriquement égale à 73,90 $\mathrm{mm}$ ) est comprise entre les extrémités de la réglette ; elle est limitée par le bord et par les deux incisions en X superposées sur une incision verticale (mesure A). Seule la face B a pu être mesurée : elle est de 73,50 $\mathrm{mm}$.

Le pouce (théoriquement égal à 24,60 mm) est compris entre les segments verticaux des faces A et B (mesures BD). Sur la face A, la mesure B est égale à 24,40 $\mathrm{mm}$ et la 
Tabl. VIII - Mesures relevées sur la réglette $n^{0} 3$ et calcul de leur erreur par rapport aux subdivisions théoriques du pes monetalis.

\begin{tabular}{|c|c|c|c|c|}
\hline \multicolumn{2}{|c|}{ RÉGLETTE N ${ }^{\circ} \mathbf{3}$} & Face A & Erreurs en mm & Erreurs en \% \\
\hline $\begin{array}{c}\text { Palmus } \\
(73,9 \mathrm{~mm})\end{array}$ & $\mathrm{A}$ & - & - & - \\
\hline \multirow{2}{*}{$\begin{array}{c}\text { Uncia } \\
(24,6 \mathrm{~mm})\end{array}$} & $\mathrm{B}$ & 24,4 & $-0,2$ & $-0,8$ \\
\cline { 2 - 5 } & $\mathrm{C}$ & 23,8 & $-0,8$ & $-3,3$ \\
\cline { 2 - 5 } & $\mathrm{D}$ & - & - & - \\
\hline $\begin{array}{c}\text { Semi-uncia } \\
(12,3 \mathrm{~mm})\end{array}$ & $\mathrm{E}$ & 12,6 & 0,3 & 2,4 \\
\hline
\end{tabular}

\begin{tabular}{|c|c|c|}
\hline Face B & Erreurs en $\mathrm{mm}$ & Erreurs en $\%$ \\
\hline 73,5 & $-0,4$ & $-0,5$ \\
\hline 24,1 & $-0,5$ & -2 \\
\hline 23,9 & $-0,7$ & $-2,8$ \\
\hline 24,5 & $-0,1$ & $-0,4$ \\
\hline 12,6 & 0,3 & 2,4 \\
\hline & 0,4 & 1,6 \\
\hline
\end{tabular}

FACE A

uncia

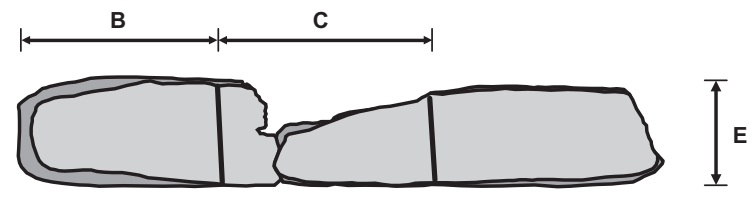

$1 / 2$ uncia

FACE B

uncia

palmus

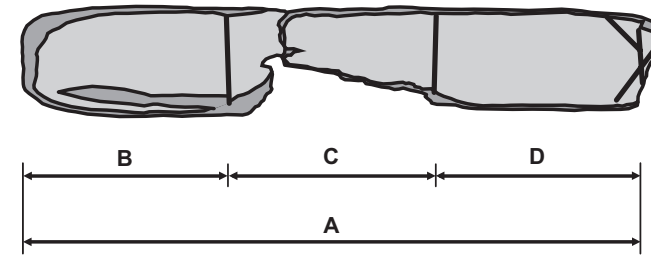

Fig. 9 - Schéma de lecture des graduations de la réglette $n^{\circ} 3$ (DAO : P. Prévot).

mesure C est égale à 23,80 mm. Sur la face B, la mesure B est égale à 24,10 mm, la mesure $\mathrm{C}$ à 23,90 $\mathrm{mm}$ et la mesure D à 24,50 mm.

Le demi-pouce (théoriquement égal à 12,30 mm) équivaut à la largeur de la réglette (mesure $\mathrm{E}$ ). Il mesure 12,60 mm sur les faces A et B.

Sans doute la réglette était étalonnée sur le demi-pied, puisqu'elle semble être brisée à sa moitié.

Il s'agit, à présent, de comparer les dimensions des graduations mesurées sur la réglette aux valeurs théoriques de la paume, du pouce et du demi-pouce, de manière à calculer le coefficient d'erreur (tabl. VIII).

Sur la face B, la paume est erronée de - 0,40 mm $(-0,5 \%)$ pour la mesure A.

Sur la face A, le pouce présente une erreur de - 0,20 mm $(-0,8 \%)$ pour la mesure B et de $-0,80 \mathrm{~mm}(-3,3 \%)$ pour la mesure C. Sur la face B, il présente une erreur de $-0,50 \mathrm{~mm}$
$(-2 \%)$ pour la mesure $\mathrm{B}$, de $-0,70 \mathrm{~mm}(-2,8 \%)$ pour la mesure $\mathrm{C}$ et de $-0,10 \mathrm{~mm}(-0,4 \%)$ pour la mesure D.

Le demi-pouce, quant à lui, présente une erreur de $0,30 \mathrm{~mm}(2,4 \%)$ pour la mesure E, sur chacune des faces de la réglette.

Il convient ensuite de déterminer le degré global de précision de la réglette $n^{\circ} 3$, en calculant la moyenne des erreurs pour chaque face. Elle est de $0,40 \mathrm{~mm}(2,2 \%)$ pour la face A et de 0,40 mm (1,6\%) pour la face B (tabl. VIII). Nous pouvons enfin déterminer la moyenne des erreurs pour l'ensemble des valeurs des faces A et B : elle est égale à $0,40 \mathrm{~mm}(1,9 \%)$. La précision des mesures relevées sur la réglette $n^{\circ} 2$, étalonnées sur la paume, le pouce et le demipouce, s'élève donc à 98,1\%.

Enfin, nous pouvons nous interroger sur la façon dont les subdivisions ont été réalisées. Pour cela, il s'agit de déterminer le pourcentage total des erreurs par type de subdivision (tabl. IX). La largeur de la réglette ne comportant qu'une graduation, nous ne calculerons que les pourcentages totaux des mesures réparties sur sa longueur.

Le pourcentage total d'erreurs de la paume, relevé sur la face $\mathrm{B}$, est de $0,5 \%$; il est de $2,2 \%$ pour le pouce, relevé sur les faces A et B.

Comme nous l'avons déjà dit, nous constatons que le pourcentage des erreurs varie de façon exponentielle (fig. 10), plus le type de mesure est petit, plus son pourcentage d'erreurs augmente. Selon nous, il s'agit d'une réglette étalonnée sur le demi-pied. Le fait que la paume ait un pourcentage d'erreurs très bas, indique qu'elle a été tracée en fonction du demi-pied qui était, lui, exact. Nous pouvons supposer que le demi-pied ait été tracé à l'aide d'un étalon. Il semble, en outre, que le pouce ait été tracé en fonction de la paume : l'inexactitude de ses dimensions par rapport à ses valeurs théoriques montre qu'il a été divisé à l'œil en trois tiers. 
Tabl. IX - Résultats du calcul de la moyenne des erreurs par types de subdivisions sur la réglette $n^{\circ} 3$.

\begin{tabular}{|l|c|c|c|}
\hline RÉGLeTte N $^{\circ} \mathbf{3}$ & Face $\mathbf{A}$ & Face $\mathbf{B}$ & Total \\
\hline Palmus & - & $0,5 \%$ & $\mathbf{0 , 5} \%$ \\
\hline Uncia & $2,1 \%$ & $1,7 \%$ & $\mathbf{2 , 2} \%$ \\
\hline
\end{tabular}

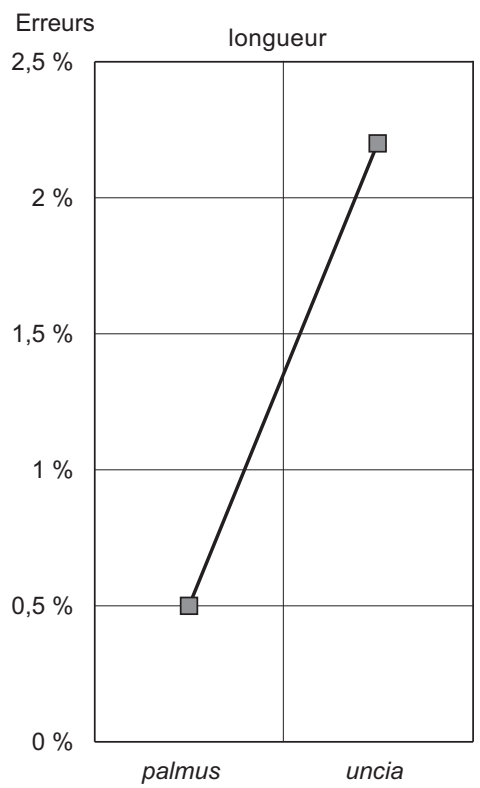

Fig. 10 - Moyenne totale des erreurs par types de mesures sur la réglette $n^{\circ} 3$ (DAO : P. Prévot).

\section{La réglette $n^{\circ} 4\left(n^{\circ}\right.$ 95.1.407 D)}

La réglette $\mathrm{n}^{\mathrm{O}} 4$ est longue de $29,90 \mathrm{~mm}$, sa largeur est de $12,10 \mathrm{~mm}$ et son épaisseur de $4,50 \mathrm{~mm}$. Nous pouvons distinguer une incision verticale sur chacune de ses faces. Les deux extrémités de la réglette ont été brisées : il semble, sur la face B, que l'une des extrémités ait été cassée sur une incision verticale.

Nous avons mesuré, sur les faces A et B, les dimensions comprises entre chaque graduation (fig. 11 et tabl. X).

Le pouce (théoriquement égal à 24,60 mm) est compris entre les segments verticaux de la face B (mesure A). La mesure A est égale à 24,50 mm.

Le demi-pouce (théoriquement égal à 12,30 mm) est égal à la largeur de la réglette (mesure B). Il mesure 12,10 mm sur les faces A et B.

Ensuite, nous pouvons comparer les dimensions des graduations relevées sur la réglette aux valeurs théoriques du pouce et du demi-pouce, afin de calculer le coefficient d'erreur (tabl. X).

Le pouce, sur la face $B$, présente une erreur de - 0,10 mm (-0,4\%) pour la mesure A.

Le demi-pouce, quant à lui, est erroné de $-0,20 \mathrm{~mm}$ $(-1,6 \%)$ pour la mesure $\mathrm{B}$ et sur chacune des faces de la réglette.

La moyenne des erreurs est de 0,20 $\mathrm{mm}(1,6 \%)$ pour la face A et de $0,20 \mathrm{~mm}(1 \%)$ pour la face B (tabl. X). Pour l'ensemble des valeurs des faces A et B, elle s'élève à $0,20 \mathrm{~mm}(1,3 \%)$ : les mesures relevées sur la réglette $\mathrm{n}^{\circ} 4$, étalonnées sur le pouce et le demi-pouce sont précises à $98,7 \%$. Ajoutons toutefois que cette précision ne porte que sur deux mesures.

\section{La réglette $n^{\circ} 5\left(n^{\circ} 95.1 .407 \mathrm{E}\right)$}

La réglette $\mathrm{n}^{\circ} 5$ est longue de $26,90 \mathrm{~mm}$, sa largeur est de $12,50 \mathrm{~mm}$ et son épaisseur varie entre $3,50 \mathrm{~mm}$ et 4,60 mm. Les deux faces ne portent pas d'incision. En revanche, seule une extrémité a été brisée.

Seul le demi-pouce a pu être repéré sur cet instrument de mesure. Il est compris dans la largeur de la réglette (fig. 12 et tabl. XI).

Il est égal à 12,50 mm en mesure A (faces A et B), alors que sa valeur théorique est de 12,30 mm.

Si nous comparons la mesure relevée à la valeur théorique du demi-pouce, nous pouvons voir qu'elle présente une erreur de 0,20 mm (1,6\%) pour les deux faces (tabl. XI).

Nous pouvons alors donner une appréciation de la précision de la réglette. Étalonnée en largeur sur le demipouce, elle est exacte à 98,6\% .

\section{INTERPRÉTATION MÉTROLOGIQUE DU LOT DE RÉGLETTES N NS $^{\text {À } 5}$}

Ce lot de réglettes semble, bel et bien, étalonné sur les subdivisions du pes monetalis. Toutefois, le manque de données relatives aux réglettes $\mathrm{n}^{\text {os }} 4$ et 5 ne nous permet pas d'interpréter ce lot d'instruments de mesure de la même manière. En effet, si l'ensemble du lot présente les mesures du doigt, en longueur, et du demi-doigt, en largeur, seules les réglettes $n^{\text {os }} 2$ et 3 paraissent étalonnées sur la paume.

Selon nous, les réglettes $n^{\text {os }} 2$ et 3 étaient à l'origine des mesures d'un demi-pied, divisées en deux paumes et six doigts sur l'ensemble de leur longueur, leur largeur étant étalonnée sur un demi-pouce. 
Tabl. X - Mesures relevées sur la réglette $n^{0} 4$ et calcul de leur erreur par rapport aux subdivisions théoriques du pes monetalis.

\begin{tabular}{|c|c|c|c|c|}
\hline \multicolumn{2}{|c|}{ RÉGLETTE N $^{\circ} \mathbf{4}$} & Face A & Erreurs en mm & Erreurs en \% \\
\hline $\begin{array}{c}\text { Uncia } \\
(24,6 \mathrm{~mm})\end{array}$ & A & - & - & - \\
\hline $\begin{array}{c}\text { Semi-uncia } \\
(12,3 \mathrm{~mm})\end{array}$ & B & 12,1 & $-0,2$ & $-1,6$ \\
\hline
\end{tabular}

\begin{tabular}{|c|c|c|}
\hline Face B & Erreurs en $\mathrm{mm}$ & Erreurs en $\%$ \\
\hline 24,5 & $-0,1$ & $-0,4$ \\
\hline 12,1 & $-0,2$ & $-1,6$ \\
\hline & 0,2 & 1 \\
\hline
\end{tabular}

Tabl. XI - Mesures relevées sur la réglette $n^{\circ} 5$ et calcul de leur erreur par rapport aux subdivisions théoriques du pes monetalis.

\begin{tabular}{|c|c|c|c|c|}
\hline \multicolumn{2}{|c|}{ RÉGLETTE N $^{\circ} \mathbf{5}$} & Face A & Erreurs en $\mathbf{~ m m}$ & Erreurs en \% \\
\hline $\begin{array}{c}\text { Semi-uncia } \\
(12,3 \mathrm{~mm})\end{array}$ & A & 12,5 & 0,2 & 1,6 \\
\hline
\end{tabular}

\begin{tabular}{c}
\begin{tabular}{|c|c|c|}
\hline Face $\mathbf{B}$ & Erreurs en $\mathbf{~ m m}$ & Erreurs en $\%$ \\
\hline 12,5 & 0,2 & 1,6 \\
\hline \multicolumn{2}{|c|}{0,2} & 1,6 \\
\hline
\end{tabular} \\
\cline { 2 - 3 }
\end{tabular}

Face A

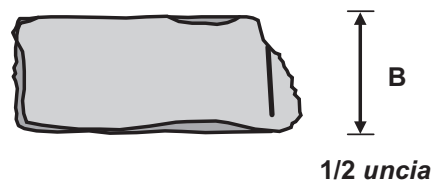

Face B

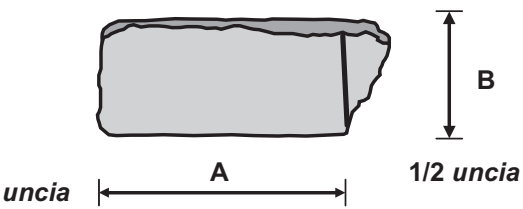

Fig. 11 - Schéma de lecture des graduations de la réglette $n^{o} 4$ (DAO : P. Prévot).
Face A

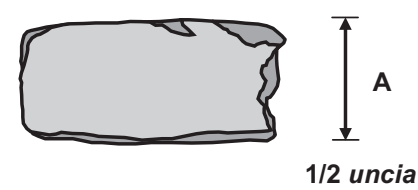

FACE B

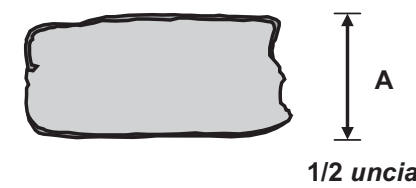

Fig. 12 - Schéma de lecture des graduations de la réglette $n^{0} 5$ (DAO : P. Prévot).
Nous pouvons néanmoins tenter de rattacher les réglettes $n^{\text {os }} 4$ et 5 aux $n^{\text {os }} 2$ et 3 en analysant et en comparant le pourcentage de précision de chacune d'entre elles (tabl. XII). L'ensemble des valeurs est compris entre $98,1 \%$ et $98,6 \%$. Nous pouvons alors dire que le pourcentage de précision des réglettes est sensiblement similaire et parler d'un lot métrologiquement homogène.

En considérant le pourcentage total d'erreurs par type de mesure et par réglette (fig. 13 et tabl. XII), nous constatons que la paume comporte $0,5 \%$ d'erreur, le pouce $1,2 \%$ et le demi-pouce $1,8 \%$. Ainsi, plus la mesure est petite plus son pourcentage d'erreurs est élevé.
Selon nous, cette répartition montre, d'une part, que les graduations des paumes comprises sur la longueur ont pu être tracées à l'œil ou avec un étalon, à partir du demipied, et celles du pouce semblent avoir été réalisées au jugé. Nous ne pouvons nous prononcer sur les graduations du demi-pouce comprises dans la largeur. Enfin, la répartition des graduations sur les réglettes paraît différer. En effet, la face $\mathrm{A}$ de la réglette $\mathrm{n}^{\circ} 2$ ne semble être marquée qu'en son centre par un $\mathrm{X}$; en revanche, sa face $\mathrm{B}$ est divisée en deux par une marque en $\mathrm{X}$ et au moins l'une des paumes possède les graduations des pouces. La réglette $n^{\circ} 3$, en face A, comporte au moins jusqu'à sa moitié les graduations du 


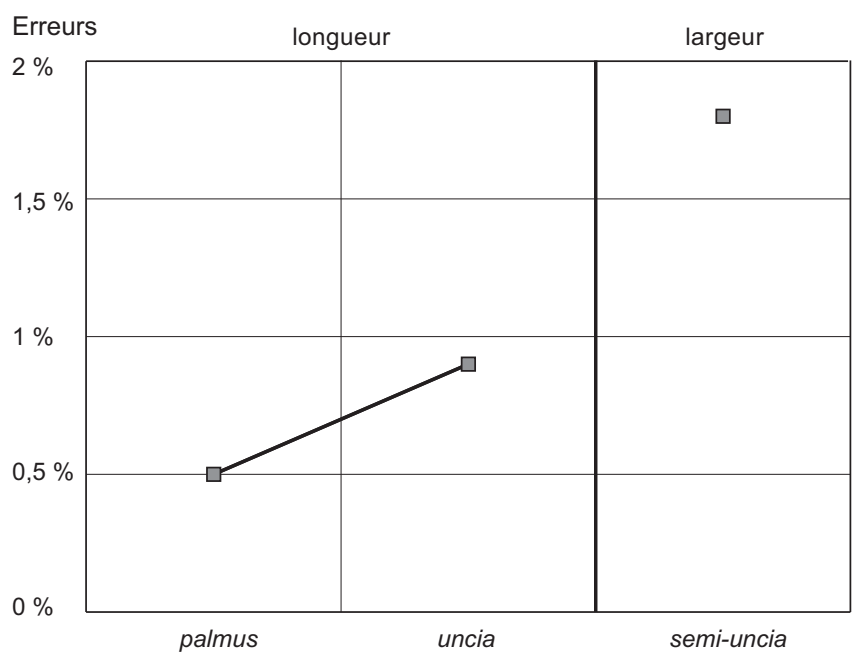

Fig. 13 - Moyenne totale des erreurs par types de mesures sur les réglettes $n^{o s} 2$ à 5 (DAO: P. Prévot).

pouce ; en face $\mathrm{B}$, elle présente, à sa moitié, un repère en $\mathrm{X}$, et au moins l'une des paumes possède les mesures du pouce. Les deux faces de la réglette $n^{\circ} 4$, comportent, au moins sur l'une de leur moitié, les subdivisions du pouce. Ainsi, nous pouvons rapprocher les réglettes $\mathrm{n}^{\text {os }} 3$ et 4 , puisque les graduations du pouce sont réparties de la même manière. En revanche, ce n'est pas le cas pour la réglette $n^{\circ} 2$.

Il est possible que la réglette $n^{\circ} 4$ soit un morceau de la $n^{\circ} 3$. L'instrument de mesure $n^{\circ} 2$, quant à lui, ne présente pas le même type de répartition pour ses graduations.

Ainsi, bien que le lot de réglettes nos 2 à 5 soit homogène par ses dimensions et ses types de subdivisions, il diffère par la répartition des graduations sur les faces des réglettes $\mathrm{n}^{\text {os }} 2$ et 3. Nous pouvons donc parler de deux types distincts.

\section{INTERPRÉTATION GÉNÉRALE DES DONNÉES DU LOT DE RÉGLETTES}

L'analyse métrologique des instruments de mesure $\mathrm{n}^{\mathrm{os}} 1$ à 5 a permis de mettre en évidence leur étalonnage sur le pes monetalis.

Toutefois, la réalisation des graduations a donné lieu à quelques erreurs de mesures. En effet, nous remarquons, de façon générale, que plus la mesure réalisée est petite, plus son rapport avec la mesure théorique est erroné. S'il est probable que ces erreurs tiennent directement de la fabrication des réglettes, ne peut-on pas penser que cela pourrait être lié, aussi, à la nécessité de précision que demande chaque type de graduations? Dans cette hypothèse, on aurait besoin de mesures très précises pour le pouce ou
Tabl. XII - Résultats du calcul de la moyenne des erreurs par types de subdivisions sur les réglettes $n^{o s} 2$ à 5 .

\begin{tabular}{|l|c|c|c|}
\cline { 2 - 4 } \multicolumn{1}{c|}{} & \multicolumn{2}{c|}{ Longueur en \% } & Largeur en \% \\
\cline { 2 - 4 } \multicolumn{1}{c|}{} & Palmus & Uncia & Semi-uncia \\
\hline RÉGLETTE N $^{\circ} \mathbf{2}$ & - & 1 & 1,6 \\
\hline RÉGLETTE N $^{\circ} \mathbf{3}$ & 0,5 & 2,2 & 2,4 \\
\hline RÉGLETTE N N $^{\circ}$ & - & 0,4 & 1,6 \\
\hline RÉGLETTE N $^{\circ} \mathbf{5}$ & - & - & 1,6 \\
\hline Total & $\mathbf{0 , 5}$ & $\mathbf{0 , 9}$ & $\mathbf{1 , 8}$ \\
\hline
\end{tabular}

\begin{tabular}{|c|}
\hline $\begin{array}{c}\text { Valeur de } \\
\text { précision en \% }\end{array}$ \\
\hline 98,5 \\
\hline 98,1 \\
\hline 98,7 \\
\hline 98,6 \\
\hline
\end{tabular}

le doigt, moins en dessous. Est-ce fonction des objets fabriqués, ou cela tient-il simplement à la plus grande difficulté du degré de précision dans la réalisation des petites subdivisions ? Les réponses à de telles interrogations, dans ce type d'étude, semblent difficiles à fournir.

Il est cependant important de noter que le degré de précision de ce lot de réglettes est remarquable, puisqu'il varie de $95 \%$ à $99 \%$. Selon nous, ces erreurs, minimes puisqu'elles sont de l'ordre du $1 / 10^{\mathrm{e}}$ de millimètres, sont dues à des graduations réalisées au jugé. Certaines mesures semblent, toutefois, avoir été effectuées à l'aide d'étalons : cela semble être le cas du demi-pied en longueur, ainsi que du doigt et peut-être du demi-pouce en largeur. L'artisan a sans doute utilisé des étalons métalliques ou des réglettes. Cela sous-entend alors l'existence et la diffusion d'étalons de mesures : peut-être des étalons officiels qui en permettaient la vulgarisation ${ }^{28}$ (Feugère, 1983). Il semble donc que ce type d'instruments participe à la romanisation par l'adoption du système métrologique romain.

Nous avons mis en évidence l'existence d'au moins trois types différents : le premier, celui de la réglette $n^{\circ} 1$ (complète), se démarquant des deux autres par des dimensions plus importantes (longueur et largeur) et des subdivisions plus précises et variées (du demi-pied au demi-doigt). Les deux autres types, ceux des réglettes $n^{\text {os }} 2$ et 3 , se différencient uniquement par la répartition des subdivisions sur leurs faces.

L'identification de ces réglettes nous permet d'établir une carte de répartition des mesures d'un pied et d'un demi-pied romain en bronze, en bois et en os (fig. 14).

Le fait que ces réglettes ont été découvertes ensemble, dans le puits de la cour $n^{\circ} 2$ (fig. 3), peut-il induire qu'elles ont été réalisées et/ou utilisées par la même personne?

28. Certaines productions de mesures pliantes du pied semblent avoir été centralisées; leur généralisation fut l'œuvre des derniers empereurs julio-claudiens. 


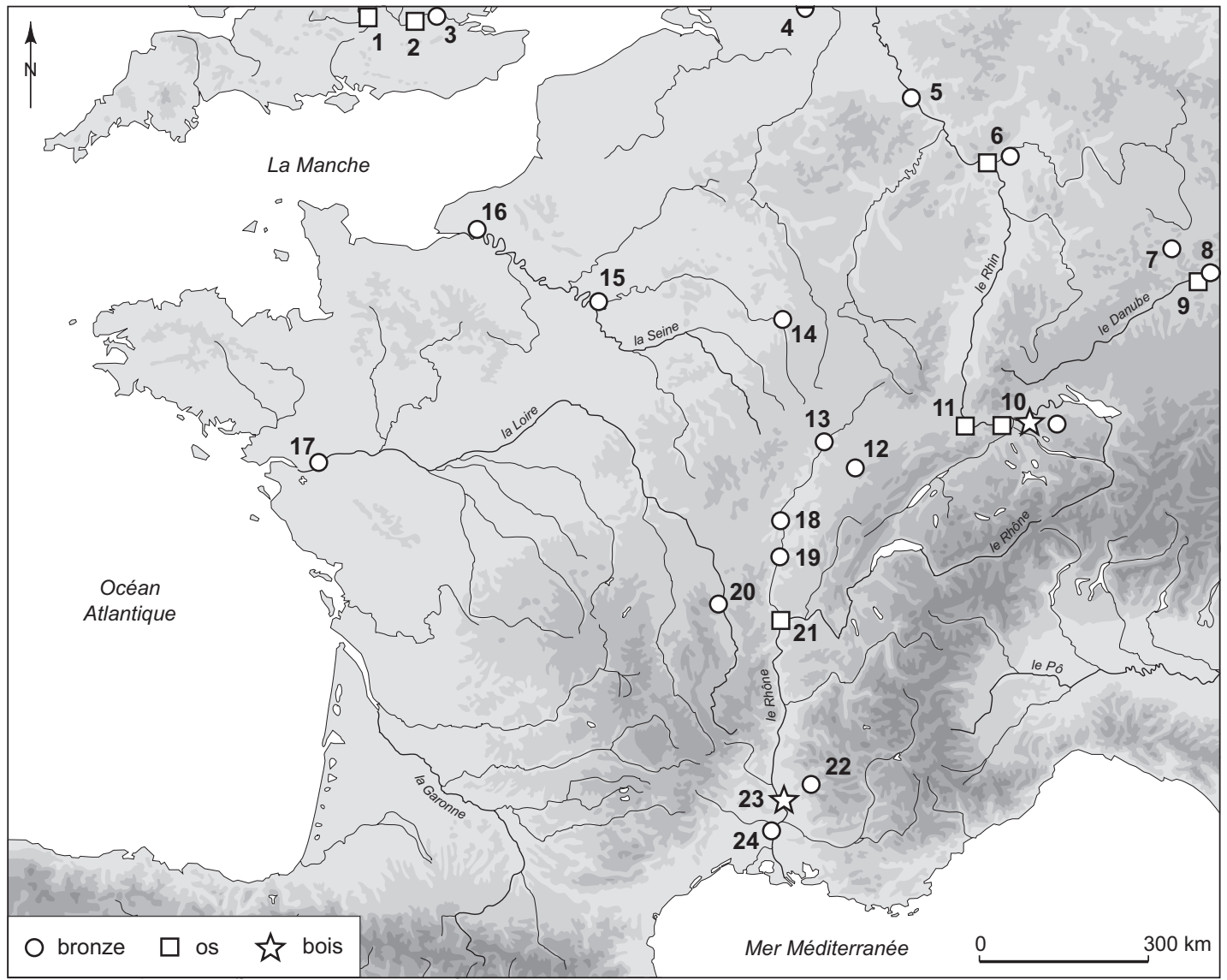

$\begin{array}{ll}\text { 1. Silchester } & \text { 7. Weissenburg } \\ \text { 2. Southwark } & \text { 8. Eining } \\ \text { 3. Londres } & \text { 9. Oberstimm } \\ \text { 4. Nimègue } & \text { 10. Vindonissa } \\ \text { 5. musée de Bonn? } & \text { 11. Augst } \\ \text { 6. Mainz } & \text { 12. Besançon (Doubs) }\end{array}$

13. Mirebeau (Vienne)

14. Bayard-sur-Marne (Haute-Marne)

15. Musée du Louvre?

16. Forêt de Maulévrier (Maine-et-Loire)

17. Mauves-sur-Loire (Loire-Atlantique)

18. Chalon-sur-Saône (Saône-et-Loire)

\begin{abstract}
19. Mâcon (Saône-et-Loire)
20. Roanne (Loire)

21. Lyon (Rhône)

22. Vaison-la-Romaine (Vaucluse)

23. Orange (Vaucluse)

24. Arles (Bouches-du-Rhône)
\end{abstract}

Fig. 14 - Répartition des mesures d'un pied et d'un demi-pied romain en bronze, en os et en bois (d'après Feugère, 1983, p. 41 fig. 2 et 1995).

Objectivement et par manque de données, la réponse reste délicate à formuler. Nous pouvons cependant noter que la présence d'un possible atelier d'assemblage d'éléments mobiliers et/ou de travail du métal à proximité (pièce $\mathrm{Z}$ ), daté de l'occupation du site entre le milieu du $\mathrm{I}^{\mathrm{er}} \mathrm{s}$. et la fin du $\mathrm{III}^{\mathrm{e}}$ s. apr. J.-C., peut avoir un rapport avec ces instruments de mesure datés, eux, terminus post quem du début du III ${ }^{\mathrm{e}}$ s. apr. J.-C. On constate, en outre, la présence d'un atelier du travail de l'os dans la domus voisine D2 (Prévot, 2008). Si les liens archéologiques entre les productions artisanales et ces instruments de mesure ne peuvent pas être établis, la présence d'ateliers comme d'outils souligne l'ambivalence de ce quartier résidentiel et artisanal.

\section{APPLICATIONS MÉTROLOGIQUES À LARTISANAT DES PETITS OBJETS ORANGEOIS}

Il semble que de tels instruments de mesure aient pu servir à la fabrication artisanale de petits objets. Nous pouvons alors nous interroger sur les rapports entre l'utilisation de ce type de réglettes et la normalisation des productions. La normalisation est-elle effectuée sur les mesures du pied romain, justifiant ainsi la présence de subdivisions précises jusqu'au demi-doigt sur les réglettes? De plus, à quel niveau de la fabrication d'un objet le calibrage intervient-il ? 

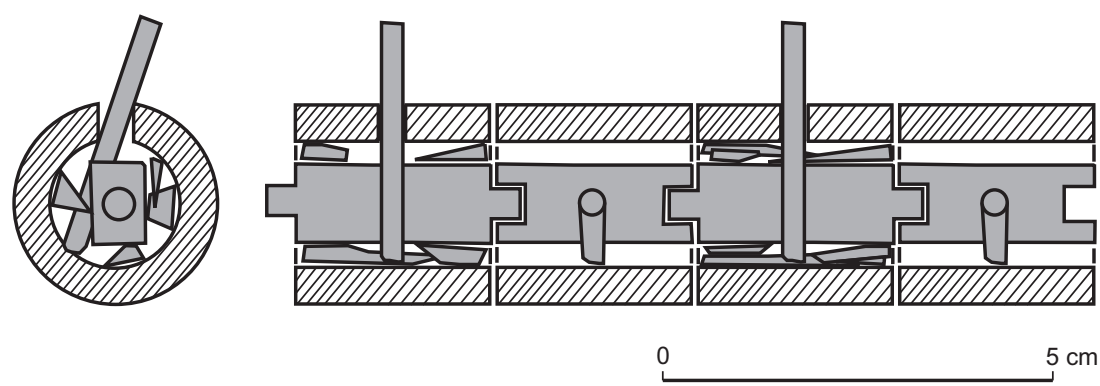

Fig. 15 - Schéma de montage des éléments de charnières en os (d'après Chabal et al., 2005).

Comme nous l'avons souligné en introduction, ce sont les objets en os, en ivoire et en bois de cerf qui semblent être les plus appropriés à la vérification de cette hypothèse. Il convient alors de réaliser une analyse métrologique sur certains types d'objets dont les mesures n'évoluent pas pendant toute la durée de leur utilisation. Il s'agit donc de trouver des séries d'objets assez importantes, produites localement et répondant à la condition de l'immobilité métrologique. Cela semble être le cas des éléments de charnières et des jetons ou pions en os issus des fouilles du site de Saint-Florent.

\section{L'EXEMPLE DE LA FABRICATION EN SÉRIE DES ÉLÉMENTS DE CHARNIÈRES EN OS}

Les éléments de charnières en os se présentent sous la forme de cylindres tournés (Fremersdorf, 1940 ; Béal, 1983 ; Prévot, 2005). Ils sont théoriquement réalisés à partir de métapodes de bovins (Barone, 1976), notamment les métatarses qui présentent la morphologie la plus adaptée à la fabrication de ce type d'objets (Prévot, 2006, fig. 27).

Ils sont de deux types distincts, établis par J.-C. Béal (1983, p. 102 et p. 110). Le type A. XI, 1 définit les éléments longs munis de deux trous latéraux ou goupilles latérales et d'une série de filets remplis de matière organique, dite matière noire à l'une de leurs extrémités. Le type A. XI, 2 regroupe, quant à lui, les éléments courts munis d'une seule goupille latérale.

Ces éléments étaient destinés à être mis bout à bout par un système de mortaises et de tenons, articulant les âmes de bois entre elles. Chaque composant, mobile et indépendant des autres, était chevillé alternativement et sur toute la longueur au dormant (le montant du meuble ou partie immobile) et à l'ouvrant (la porte du meuble ou partie mobile) (fig. 15).

Si des interrogations persistent sur la façon dont le tournage de ces cylindres était réalisé, à savoir en série ou non, il semble qu'une homogénéité des diamètres ait été nécessaire au montage. Le tournage, pour une même série d'éléments de charnières et quel qu'en soit le type, devait permettre de produire des diamètres égaux au millimètre près de manière à faciliter le montage et le fonctionnement, voire également l'esthétique, du dispositif.

La longueur de chaque élément, quant à elle, était calculée ${ }^{29}$ en fonction du nombre de charnières à placer sur le dispositif et selon la longueur du montant. Ainsi, elle peut varier fortement d'une série à une autre, mais reste homogène par type sur le même dispositif.

Il semble enfin qu'il n'y ait pas de rapport entre leur diamètre et leur longueur. Sur des systèmes de charnières différents, si les diamètres varient généralement peu, les longueurs restent, quant à elles, propres à chaque dispositif.

Ainsi, compte tenu des critères techniques que nous venons d'exposer, nous analyserons, d'une part, l'ensemble des diamètres de la totalité des éléments de charnières du site de Saint-Florent ${ }^{30}$, tous types confondus, de façon à voir s'ils ont été tournés à partir des subdivisions du pied et donc à partir d'étalons, et, d'autre part, nous étudierons les longueurs des deux séries les plus importantes du site ${ }^{31}$. Notre étude du calibrage de la longueur ne prenant en compte que les éléments de type A. XI, 2 de chacune des deux séries ${ }^{32}$.

\section{ÉTUDE DES DIAMÈTRES}

Nous avons relevé les diamètres de l'ensemble des éléments de charnières, soit 78 objets provenant du site de Saint-Florent (tabl. XIII) et les avons représentés sur

29. La calibrage en hauteur est réalisé entre les étapes de sélection de la zone utile et du tournage.

30. Séries $n^{\text {os }}$ 95.1.377-387 et 543-544.

31. Séries $n^{\text {os } 95.1 .377 ~ e t ~} 543$.

32. Les éléments courts étant les plus nombreux sur un système de charnières. 


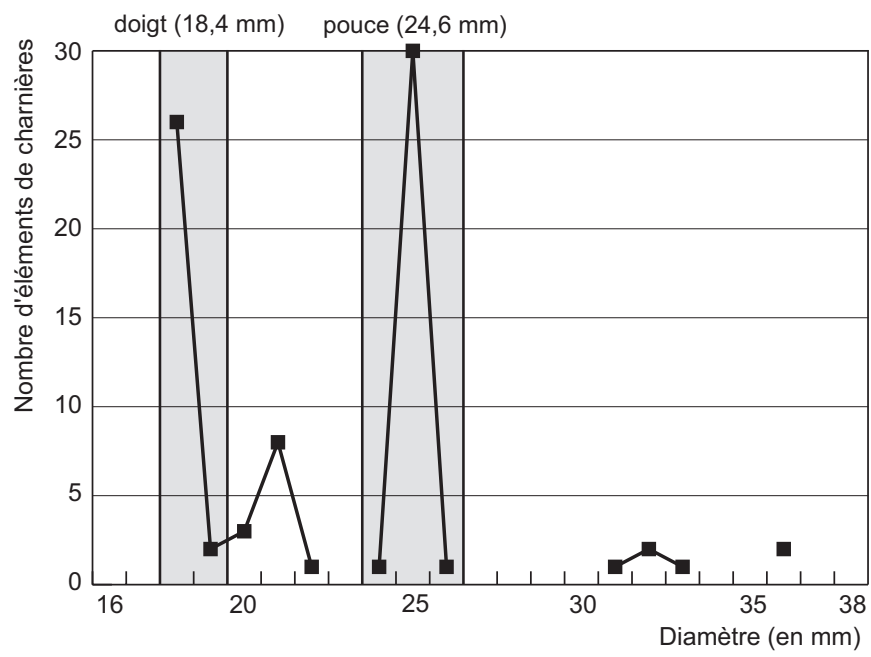

Fig. 16 - Répartition des diamètres relevés sur les éléments de charnières en os provenant de Saint-Florent à Orange : en grisé, les correspondances sur les mesures du doigt et du pouce (DAO : P. Prévot).

le graphique de la figure 16, après les avoir regroupés par classes de mesures.

Nous remarquons deux pics principaux de mesures (fig. 16). Le premier, étalonné sur la classe de mesure « $18 \mathrm{~mm}$ », comprend 26 éléments de charnières. Le second, étalonné sur la classe de mesure " $25 \mathrm{~mm}$ ", compte 30 objets. Il est intéressant de constater que ces deux pics statistiques sont étalonnés sur des classes de mesures qui se rapprochent des dimensions du doigt $(18,40 \mathrm{~mm})$ et du pouce $(24,60 \mathrm{~mm})$.

Le calcul de l'erreur moyenne à partir du nombre d'unités des classes de mesures les plus proches du doigt et du pouce peut permettre de les comparer à celles identifiées sur les réglettes $\mathrm{n}^{\text {os }} 1$ à 4 . L'erreur moyenne des diamètres calibrés sur le pouce est de $0,1 \mathrm{~mm}$, soit $0,2 \%$; celle des diamètres étalonnés sur le doigt est de $0,10 \mathrm{~mm}$, soit $0,4 \%$. Nous constatons la relative précision du calibrage dont le pourcentage d'erreurs est quasi nul. Selon nous, il semble que pour $72 \%$ des éléments de charnières, mis au jour à Saint-Florent, le calibrage des diamètres ait été réalisé et étalonné sur les mesures du pouce et du doigt. Comme nous l'avons souligné plus haut, l'étalonnage sur des mesures précises répond à un impératif technique de montage et de fonctionnement.

Enfin, nous pouvons comparer les pourcentages moyens d'erreurs des diamètres étalonnés sur les subdivisions du pes monetalis aux pourcentages moyens d'erreurs relevés sur les réglettes de la domus E2. Les mesures du pouce, visibles sur les réglettes $\mathrm{n}^{\text {os }} 1$ et 2-4, présentent respectivement une erreur moyenne de $4,3 \%$ et $1,2 \%$. Les mesures du doigt,
Tabl. XIII - Diamètres, par classes de mesures, des éléments de charnières en os provenant de Saint-Florent à Orange.

\begin{tabular}{|c|c|}
\hline Diamètre en mm & Nombre de charnières \\
\hline 16 & 0 \\
\hline 17 & 0 \\
\hline 18 & 26 \\
\hline 19 & 2 \\
\hline 20 & 3 \\
\hline 21 & 8 \\
\hline 22 & 1 \\
\hline 23 & 0 \\
\hline 24 & 1 \\
\hline 25 & 30 \\
\hline 26 & 1 \\
\hline 27 & 0 \\
\hline 28 & 0 \\
\hline 29 & 0 \\
\hline 30 & 0 \\
\hline 31 & 1 \\
\hline 32 & 2 \\
\hline 33 & 1 \\
\hline 34 & 0 \\
\hline 35 & 0 \\
\hline 36 & 2 \\
\hline 37 & 0 \\
\hline 38 & 0 \\
\hline Total & 78 \\
\hline
\end{tabular}

seulement visibles sur la réglette $\mathrm{n}^{\mathrm{o}} 1$, sont erronées à $2,7 \%$ en moyenne. Bien que les erreurs moyennes du pouce, sur les séries de charnières et les réglettes, varient entre $0,2 \%$ et $4,3 \%$ et que celles du doigt varient entre $0,4 \%$ et $2,7 \%$, ces pourcentages équivalent à des dixièmes de millimètres. Nous constatons alors la précision de l'étalonnage des mesures à la fois sur des outils et sur des productions. Ainsi, d'un point de vue métrologique, nous pouvons faire le lien entre les deux. Cependant, rien n'indique le lien direct de ces réglettes avec la production de ces éléments de charnières eux-mêmes. Pour des besoins évidents de précision, les artisans ont dû avoir recours, durant l'étape du tournage, à des étalons de mesure semblables aux réglettes dont nous avons réalisé l'étude.

\section{ÉTUDE DES LONGUEURS}

Il s'agit à présent d'étudier la répartition des longueurs des éléments de charnières de deux séries provenant, 
Tabl. XIV - Longueurs, par classes de mesures, des éléments de charnières en os provenant de Saint-Florent à Orange.

\begin{tabular}{|c|c|c|}
\cline { 2 - 3 } \multicolumn{1}{c|}{} & \multicolumn{2}{c|}{ Série d'éléments de charnières } \\
\hline Longueur en $\mathbf{~ m m}$ & $\mathbf{n}^{\circ} \mathbf{9 5 . 1 . 3 7 7}$ & $\mathbf{n}^{\circ} \mathbf{9 5 . 1 . 5 4 3}$ \\
\hline 23 & 0 & 0 \\
\hline 24 & 1 & 0 \\
\hline 25 & 4 & 1 \\
\hline 26 & 8 & 8 \\
\hline 27 & 7 & 7 \\
\hline 28 & 0 & 3 \\
\hline 29 & 1 & 0 \\
\hline 30 & 0 & 0 \\
\hline Total & $\mathbf{2 1}$ & $\mathbf{1 9}$ \\
\hline
\end{tabular}

également, du site de Saint-Florent. La série $\mathrm{n}^{\circ}$ 95.1.377 compte 21 éléments, alors que la série $\mathrm{n}^{\circ} 95.1 .543$ en compte 19. Nous ne prenons en compte que les éléments de type $\mathrm{A}$. XI, 2, théoriquement homogène du point de vue typologique.

Nous avons regroupé l'ensemble des données par classes de mesures (tabl. XIV). Il apparaît, pour la série $\mathrm{n}^{\mathrm{o}}$ 95.1.377, que la plupart des longueurs sont réparties entre les classes «24-27 $\mathrm{mm}$ ». Les longueurs de la série $\mathrm{n}^{\mathrm{0}}$ 95.1.543, quant à elles, sont comprises entre les classes « 25-28 mm ». Dans les deux cas, les classes les plus denses sont les « 26-27 mm » (fig. 17, $\mathrm{n}^{\text {os }} 1$ et 2).

Nous remarquons que la quasi-totalité des longueurs varie à proximité de la mesure du pouce $(24,60 \mathrm{~mm})$, soit de façon exacte, soit légèrement au-dessus. L'erreur moyenne des longueurs vraisemblablement calibrées sur le pouce est de $2,10 \mathrm{~mm}$, soit $8,3 \%$, pour les éléments de la série $\mathrm{n}^{\circ} 95.1377$; elle est de $2,60 \mathrm{~mm}$, soit $10,7 \%$, pour les charnières de la série $\mathrm{n}^{\circ} 95.1 .543$. Nous constatons, premièrement, que ces séries sont très homogènes, bien que provenant de contextes archéologiques différents: il est probable que les gabarits des meubles auxquels elles appartenaient étaient les mêmes. Deuxièmement, il semble que les longueurs de la majorité des éléments de type A. XI, 2 aient été calibrées sur le pouce.

La variation des erreurs par rapport à la mesure du pouce paraît attester une marge de manœuvre plus importante concernant le calibrage en longueur des éléments de charnières. Elles ont probablement été calibrées de façon moins précise que les diamètres, car il est toujours possible, en cas d'erreur, d'ajuster le dernier élément du dispositif à la longueur du montant. Ce n'est pas le cas des diamètres, difficilement modulables par un nouveau tournage.
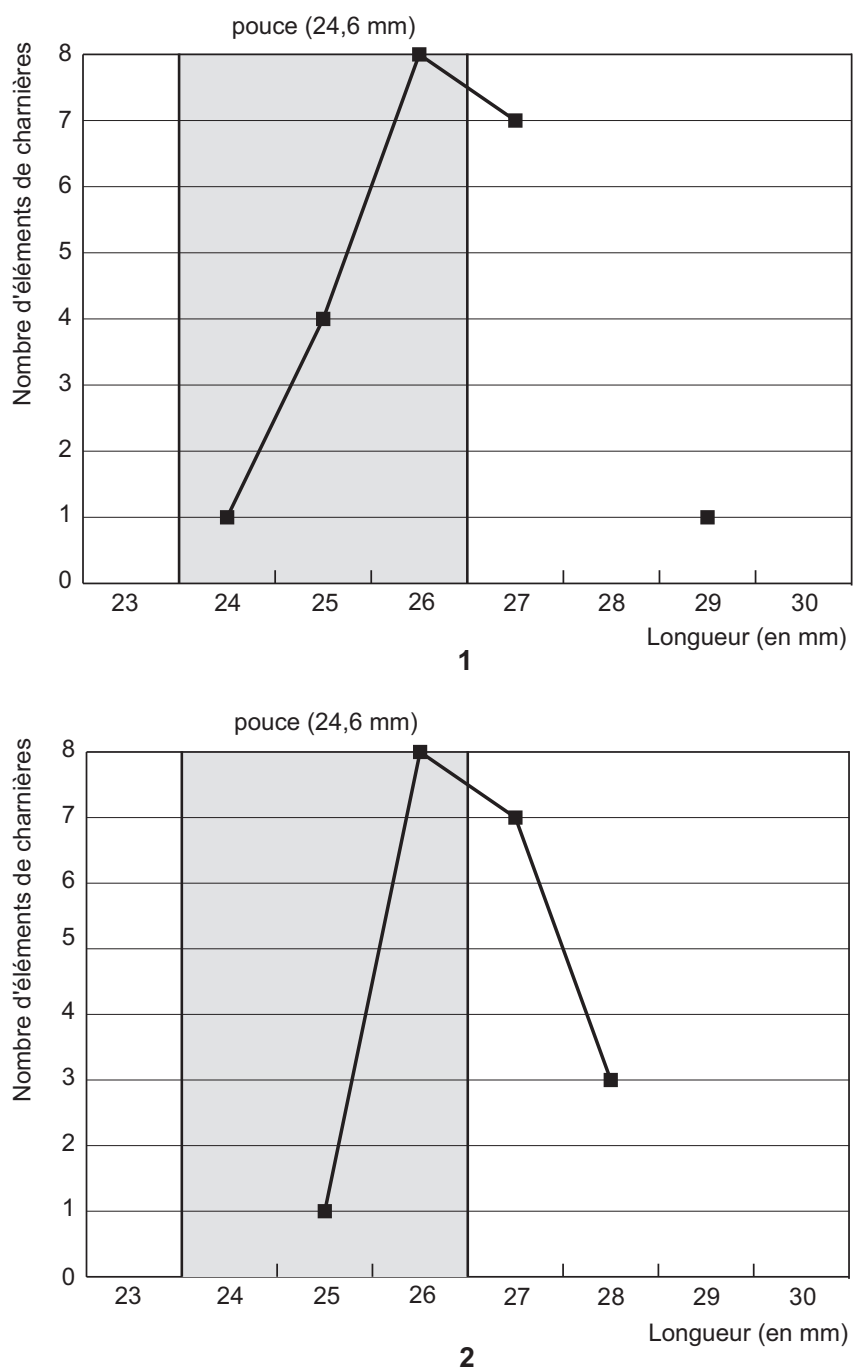

Fig. 17 - Répartition des longueurs relevées sur les éléments de charnières en os provenant de Saint-Florent à Orange : 1, série $n^{\circ}$ 95-1377 ; 2, série $n^{o}$ 95-1-543 ; en grisé, les correspondances sur la mesure du pouce (DAO : P. Prévot).

Il est donc parfaitement envisageable qu'un étalonnage sur le pouce, au moyen d'instruments de mesure, ait été réalisé sur ces éléments de charnières.

\section{NORMALISATION DE LA PRODUCTION DES JETONS}

Dans un second temps, il convient de s'attarder sur les jetons ou pions en os. Ils avaient des fonctions très précises dans les jeux de table romains et tenaient une place relativement importante dans les divertissements de l'époque (Béal, Dureuil, 1996, p. 95-99). On les trouve en général en grand nombre dans les zones d'habitat et commerciales 
Tabl. XV - Diamètres, par classes de mesures, des jetons ou pions en os provenant de Saint-Florent à Orange.

\begin{tabular}{|c|c|}
\hline Diamètre en $\mathbf{~ m m}$ & Nombre de jetons \\
\hline 10 & 0 \\
\hline 11 & 1 \\
\hline 12 & 1 \\
\hline 13 & 0 \\
\hline 14 & 1 \\
\hline 15 & 4 \\
\hline 16 & 0 \\
\hline 17 & 2 \\
\hline 18 & 6 \\
\hline 19 & 1 \\
\hline 20 & 6 \\
\hline 21 & 0 \\
\hline & $\mathbf{2 2}$ \\
\hline
\end{tabular}

situées en contexte urbain. Outre le jeu, les jetons ou pions pouvaient être des supports de comptes pour les activités commerciales (Luik, 1994 ; Schärlig, 2003), mais également pour les jeux de hasard.

Plusieurs méthodes de fabrication des jetons sont connues à l'époque antique. Dans tous les cas de figure, l'artisan doit prélever au départ une plaquette (généralement sur des scapulas de bovins). Les modes de débitage des jetons peuvent différer ensuite selon les ateliers. L'artisan pouvait prélever des petits carrés d'os dans les plaquettes qu'il fixait sur un tour, entre une poupée et un pointeau. Il tournait ensuite le jeton afin de lui donner la forme d'un disque. Il pouvait aussi fixer directement la plaquette sur un tour et extraire les jetons de la matrice par pression ou à l'aide d'un outil tranchant. Ces deux façons de procéder sont assez similaires. Une troisième et dernière méthode consiste en l'extraction des jetons à l'aide d'un outil rotatif. Ce procédé de fabrication a été mis en évidence grâce aux rebuts de tournage comportant des trous perforants. Les jetons étaient vraisemblablement extraits de la matrice à l'aide d'une scie cloche rotative, comme le montrent les parois à profil vertical, visibles sur les matrices. Ce dispositif d'extraction était sans doute similaire à celui retrouvé à Bingen (Allemagne) (Künzl, 1983, p. 20-21 et p. 84-85), dans une tombe de médecin, datée de la fin du $\mathrm{I}^{\mathrm{er}} \mathrm{s}$. ou du début du II $^{\mathrm{e}}$ s. apr. J.-C. ${ }^{33}$ Enfin, il n'est pas impossible que

33. Des instruments médicaux de trépanation ont été identifiés : il s'agit de deux scies cloches en bronze de diamètre complémentaire que le médecin faisait tourner à l'aide d'un archet pliant en bronze.

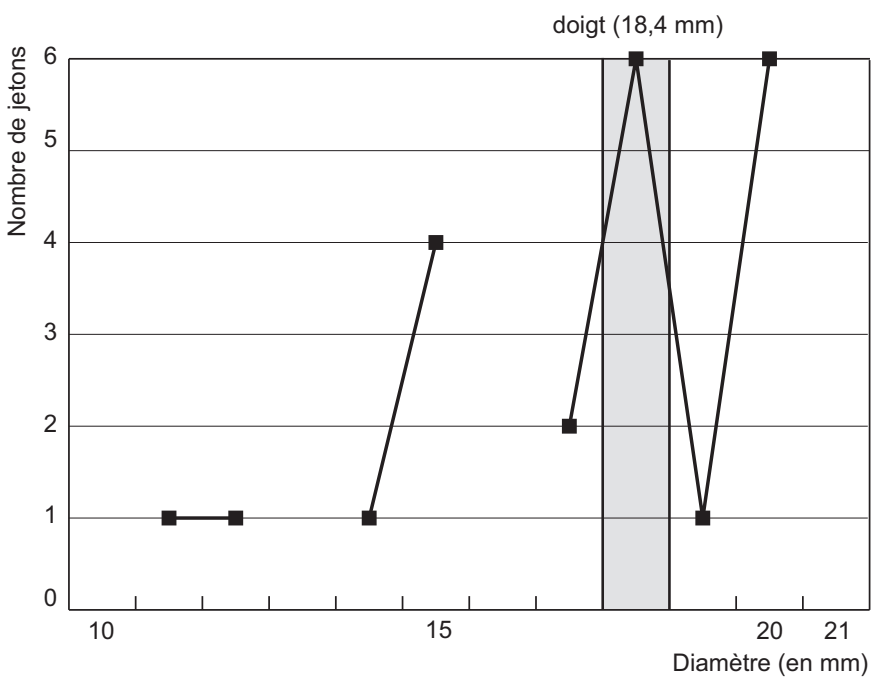

Fig. 18 - Répartition des diamètres relevés sur les jetons ou pions en os provenant de Saint-Florent à Orange : en grisé, les correspondances sur la mesure du doigt (DAO : P. Prévot).

l'on ait eu recours à des compas à deux pointes, dont un tranchant, pour extraire les jetons de cette manière.

Pour terminer, les ébauches de jetons étaient finalisées et décorées ou non par tournage ou polissage.

Premièrement, nous pouvons nous attarder sur les diamètres des 22 jetons ou pions ${ }^{34}$ issus des fouilles du site de Saint-Florent (tabl. XV) : cette série étant, à l'échelle orangeoise, la plus importante. Ces jetons semblent être étalonnés sur trois types de diamètres principaux (fig. 18). Il s'agit des classes de mesures « $15 \mathrm{~mm}$ », « $18 \mathrm{~mm}$ » et « $20 \mathrm{~mm}$ ». La série « $18 \mathrm{~mm}$ » est la plus dense, avec 6 jetons. Cette dernière se rapproche de la mesure théorique du doigt. L'erreur moyenne des diamètres potentiellement calibrés sur le doigt, à savoir les classes de mesures «17$20 \mathrm{~mm}$ " qui regroupent près de 14 jetons ou pions, est de $1 \mathrm{~mm}$, soit 5,4\%. Le fait que $64 \%$ de la totalité des pions ou jetons tournent autour de la valeur théorique du doigt, à $1 \mathrm{~mm}$ d'erreur près, paraît significatif d'un étalonnage sur cette mesure.

Deuxièmement, l'étude des matrices de jetons ou de pions retrouvées dans l'atelier du travail de l'os de la maison E1 35 (Prévot, 2008) a montré que sur les 66 exemplaires

Un trou de pointage permettait de caler la cloche sur le crâne du patient et de faire pivoter l'outil qui sciait, ainsi, la face externe du crâne. Il est probable que les artisans de l'os aient pu recourir à une technique similaire pour extraire les jetons des plaquettes.

34. Jetons $n^{\text {os }} 95.1 .193,350-370$ et 423.

35. Cet atelier est daté de l'état I du site, soit entre 15-10 av. J.-C. et 10-15 apr. J.-C. 


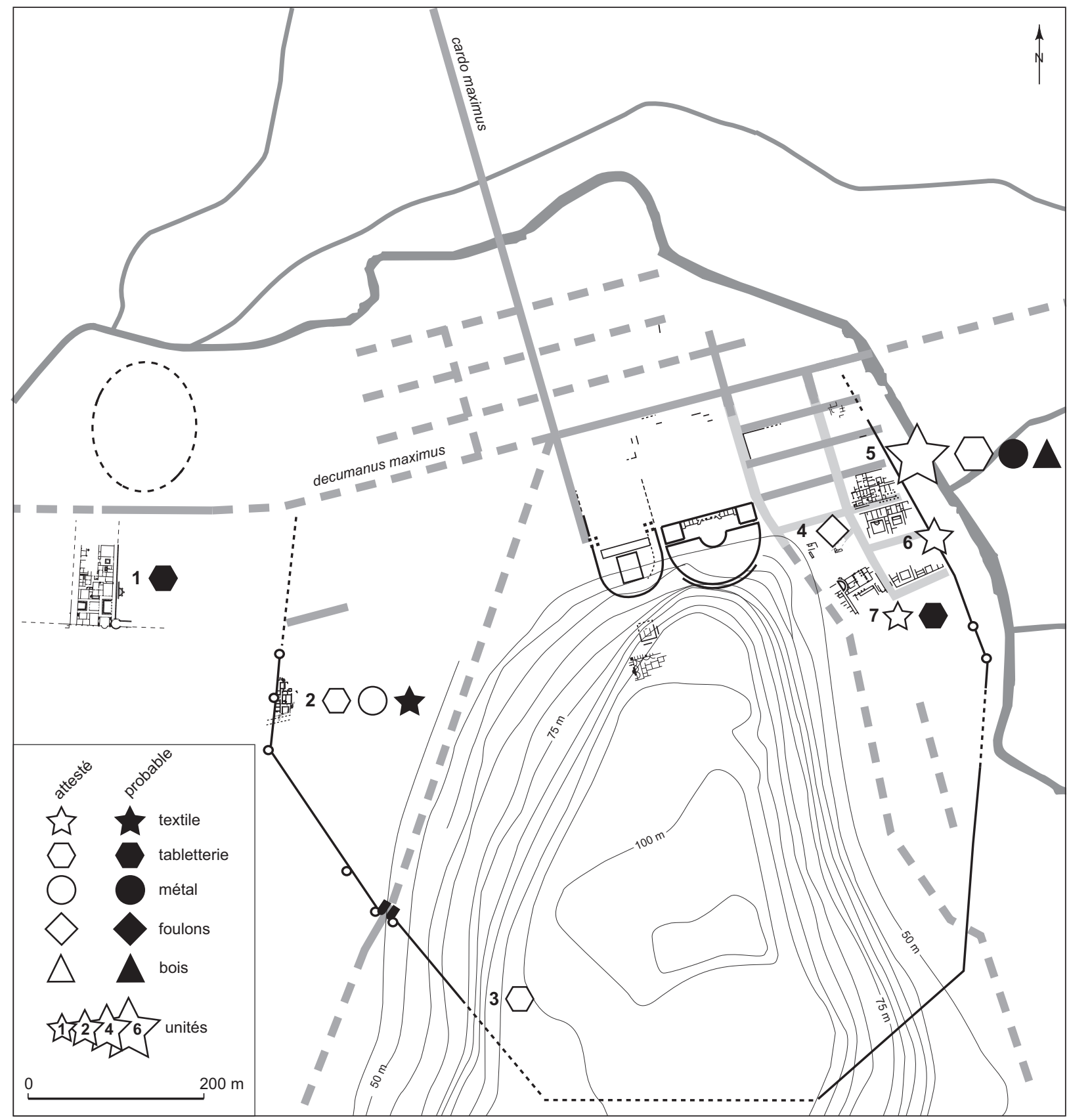

\begin{tabular}{|c|c|c|c|}
\hline Site & 20-15 av. / 15-20 apr. J.-C. & 20-30 apr. / II s. apr. J.-C. & fin $\|^{\mathrm{e}}$ s. I III $\mathrm{e}$. apr. J.-C. \\
\hline 1. La Brunette & & 1 artisanat de l'os & \\
\hline 2. Le Mas des Thermes & & 1 artisanat du métal & $\begin{array}{l}1 \text { artisanat de l'os; } \\
1 \text { artisanat du textile? }\end{array}$ \\
\hline 3. Chemin de la Maguelonne & & 1 artisanat de l'os & \\
\hline 4. L'Hôpital & & 1 foulonnerie & \\
\hline 5. Saint-Florent, îlot nord & $\begin{array}{l}4 \text { artisanats domestiques du textile; } \\
1 \text { artisanat de l'os }\end{array}$ & \multicolumn{2}{|c|}{$\begin{array}{l}2 \text { artisanats domestiques du textile ; } 1 \text { artisanat de l'os ; } \\
1 \text { artisanat du bois ? ; } 1 \text { artisanat du métal ? }\end{array}$} \\
\hline 6. Saint-Florent, îlot sud & & \multicolumn{2}{|l|}{2 artisanats domestiques du textile } \\
\hline 7. Cours Pourtoules & & 1 artisanat domestique du textile & 1 artisanat de l'os? \\
\hline
\end{tabular}

Fig. 19 - Répartition des vestiges d'artisanat attestés ou probables à Orange, datés du dernier quart du I'r s. av.J.-C. au III $s$. apr. J.-C. (d'après fond de carte C. Allinne; DAO : P. Prévot et J.-M. Mignon, SADV). 
retrouvés ${ }^{36}, 52$ présentent un trou d'extraction de $19 \mathrm{~mm}$, 7 de 21,50 mm, 6 de 23,50 mm et 1 de $31 \mathrm{~mm}$. En admettant qu'il y ait un décalage minimum d'environ $1 \mathrm{~mm}$, correspondant au tranchant de l'outil d'extraction, nous pouvons rapprocher les matrices calibrées sur $19 \mathrm{~mm}$ de la mesure du doigt.

Il semble que la production d'une partie de certains jetons ou pions en os ait été calibrée sur le doigt, cette mesure de référence permettant certainement de calibrer la production et de la réaliser en série. Il semble s'agir alors d'une commodité technique, vraisemblablement réalisée à l'aide d'un étalon de référence.

\section{NORMALISATION DES PRODUCTIONS EN SÉRIE EN OS}

L'étude et l'analyse métrologique de ces séries d'objets en os, issus du site de Saint-Florent, a permis de mettre en évidence l'étalonnage d'une partie des productions sur certaines subdivisions du pes monetalis.

En effet, il semble que la plupart des diamètres des éléments de charnières en os aient été calibrés sur le pouce et le doigt. Les hauteurs, quant à elles, paraissent avoir été étalonnées sur le pouce. Des raisons techniques requérant une relative précision pour le montage et le fonctionnement de ces éléments semblent être à l'origine des calibrages.

Dans le cas des diamètres des pions ou jetons en os, nous avons constaté qu'un calibrage autour de la valeur du doigt avait été effectué. Il semble que cet étalonnage soit le fait d'une commodité technique visant à réaliser des productions sérielles de diamètres identiques. Dans ce cas, il paraît plus facile de se calquer sur une mesure de référence.

La normalisation des productions sérielles en os semble passer par l'emploi d'étalons de mesures semblables à ceux de la maison E2. Il semble que la production en série d'objets manufacturés en os, qui répondent à des critères typologiquement propres, ne puisse être dissociée de l'emploi d'instruments de mesure précis.

36. Il s'agit des exemplaires nos 95.1.234 A-R, 236, 237, 239, 240, 242 A-B, 243 A-H, 244 A-Z, 246 A et C et 245 A-F.
À l'échelle du site de Saint-Florent, le manque d'informations archéologiques ne nous a pas permis de mettre en relation, de manière précise, ces outils et la domus E2. Toutefois, la présence dans le puits de fuseaux qui semblent montrer qu'un artisanat lié au textile y a été pratiqué, ou encore la présumée activité artisanale liée au métal et au montage de placages décoratifs sur des éléments mobiliers, repérée dans la pièce $\mathrm{Z}$, pourraient être des indices du contexte d'utilisation de ces réglettes. Elles sont, quoi qu'il en soit, des indicateurs de l'utilisation de ce type d'outils dans le contexte artisanal orangeois (fig. 19).

Au-delà du caractère exceptionnel de ce type d'instrument de mesure, l'analyse et l'étude technique des réglettes $\mathrm{n}^{\text {os }} 1$ à 5 nous ont permis de démontrer, d'une part, leur étalonnage sur le semi-pes monetalis et ses subdivisions et, d'autre part, la possible diffusion, par ce type d'instrument de mesure, d'étalons métrologiques. La reproduction et la diffusion d'étalons permettaient, peut-être, de romaniser la Gaule et d'unifier le système métrologique de manière à mieux le contrôler.

En outre, il semble effectivement que des instruments de mesure de ce type aient pu être utilisés dans les processus de fabrication de petits objets, nécessitant des étalons de mesure précis, comme le pouce, le demi-pouce, le doigt et le demi-doigt. Selon nous, les caractères morphologiques de certains types d'objets, et plus particulièrement ceux du petit mobilier en os pourraient attester une large utilisation et diffusion de ces mesures. Il semblerait donc que ce type de réglettes participe à la normalisation technique du travail ${ }^{37}$ et permette d'étalonner les productions en fonction de critères technologiques ${ }^{38}$.

37. Il est probable, par exemple, dans un souci technique, que des instruments de mesure aient permis d'étalonner des pyxides à leur couvercle ou vice versa.

38. Je tiens à remercier J.-M. Mignon (Service d'archéologie du département du Vaucluse) pour avoir accepté de me confier l'étude de ce lot, ainsi que X. Lafon (Université de Provence, IRAA, UMR 6222 du CNRS), M. Feugère (équipe TPC, UMR 5140 du CNRS) et P. Borgard (CCJ, UMR 6573 du CNRS) pour leur aide, conseils et relectures à différents degrés. 


\section{BIBLIOGRAPHIE}

\section{ANDERSON J. C. Jr}

2001: "Anachronism in the Roman Architecture of Gaul: the Date of the Maison Carrée at Nîmes ", The Journal of the Society of Architectural Historians, 60, p. $68-79$

\section{BARONE R.}

1976 : Anatomie comparée des mammifères domestiques, $2^{\mathrm{e}}$ éd. revue et mise à jour, Paris, Vigot Frères.

\section{BARTHÉLEMY D., DUbois S.}

2007 : «Métrologie agraire antique : une tige métallique graduée découverte à Mâcon (Saône-et-Loire) ", Revue archéologique de l'Est, 56, p. 371-379.

BÉAL J.-C.

1983 : Catalogue des objets de tabletterie du musée de la Civilisation gallo-romaine de Lyon, Lyon, De Boccard, 421 p.

BÉAL J.-C., DUREUIL J.-F.

1996 : La Tabletterie gallo-romaine et médiévale : une histoire d'os, Paris, Paris Musées (coll. Catalogue d'art et d'histoire du musée Carnavalet, XI), 123 p.

\section{BRIDGER C. J.}

1984 : « The Pes Monetalisand the PesDrusianus in Xanten », Britannia, 15, p. 85-98.

\section{Chabal L., Feugère M.}

2005: «Le mobilier organique des puits antiques et autres contextes humides de Lattara ", in BUXó R., PIQUÈs G. (DIR.), Onze puits gallo-romains ( $e^{e r} s . a v$.

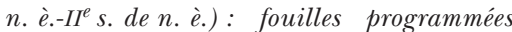
1986-2000, Lattes (coll. Lattara, 18), p. $137-188$.

\section{Chenorkian R.}

1996: Pratique archéologique, statistique et graphique, Paris, Errance-ADAM, 162 p.

Daremberg C., Saglio E., Pottier E. (DIR.)

1877-1919 : Dictionnaire des Antiquités grecques et romaines d'après les textes et monuments, Paris, Hachette, 10 vol.

\section{DUNCAN-JONES R. P.}

1980 : "Length-Units in Roman Town Planning: the Pes Monetalis and the Pes Drusianus », Britannia, 11, p. 127-133.
Faure V., Gasciou J., Mignon J.-M., PLANCHON J., ZUGMEYER S.

1999 : «Un sévir augustal d'Orange et de Lyon ", Revue archéologique de Narbonnaise, 32, p. 21-30.

\section{FELLMANN R.}

1988 : La Suisse gallo-romaine: cinq siècles d'Histoire, Stuttgart, Konrad Theiss Verlag GmbH \& Co., 470 p. (rééd. Lausanne, Payot, 1992).

\section{FEUGÈre M.}

1983 : « Les mesures pliantes du pied romain en bronze et en os : à propos d'un exemplaire conservé à Roanne », Cahiers archéologiques de la Loire, 3, p. 39-43. chets : le monde des petits objets ", in GUILHOT J.-O., GOY C. (DIR.), $20000 \mathrm{~m}^{3}$ d'histoire : les fouilles du parking de la Mairie à Besançon, Besançon, Musée des BeauxArts et d'Archéologie, p. 130-171.

1994 : «Les fouilles du parking de la Mairie de Besançon : petits objets des niveaux laténiens ", in JUD P. (DIR.), Die spätkeltische Zeit am südlichen Oberrhein/Le Rhin supérieur à la fin de lépoque celtique, Actes du colloque de Bâle, 1991, Bâle, Archäologische Bodenforschung des Kantons BaselStadt, p. 146-155.

1995 : «Une mesure d'un demi-pied romain à Chalon-sur-Saône (Saône-et-Loire)", Revue archéologique de l'Est, 46/1, p. 151153

\section{FREMERSDORF F.}

1940 : «Römische Scharnierbänder aus Bein ", in Festchrift Victor Hoffiller (Serta Hoffileriana), Zagreb, Aufsätze der röm. germ. Abteilung des Wallrafrichartz Museums der Hansestadt Köln, p. 321-337.

\section{GOSTENČNIK K.}

1998: "Römische Fußmaßstäbe vom Magdalensberg », Carinthia, I, 188, p. $87-107$.

\section{HÉRON DE VILLEFOSSE A.}

1902: Outils d'artisans romains, Nogentle-Rotrou, Imprimerie Daupeley Gouverneur (coll. Mémoires de la Société des Antiquaires de France, 63), 335 p.

1907 : s. v. «Pes", in DAREMBerG C., SAGLIO E., POTTIER E. (DIR.), Dictionnaire
1992 : «Bibelots, quincaillerie et colifi- des Antiquités grecques et romaines, IV/1, Paris, Hachette, p. 419-421.

\section{HULTSCH F}

1882: Griechische und römische Metrologie, Berlin, Weidmann (rééd. Graz, 1971).

\section{HUSSONG L.}

1938: «Ein Brandgrab mit Bronzemastab um 300 n. Chr. ", in Festschrift A. Oxé, Darmstadt.

\section{HYGIN}

1848: «De condicionibus agrorum », in LACHMANn C. (DIR.), Oromatici Veteres, t. I, Berlin, Bei Georg Reimer, 416 p.

\section{KIDSON P.}

1990 : « A Metrological Investigation », Journal of the Warburg and Courtauld Institutes, 53 , p. 71-97.

\section{KÜNZL E.}

1983: Medizinische Instrumente aus Sepulkralfunden der römischen Kaiserzeit, Bonn, Rheinisches Landesmuseum Bonn.

\section{LUIK M.}

1994 : « Ein Körpergrab mit Spielsteinbeigabe aus Köngen, Kries Esslingen ", Fundber aus Baden-Württemberg, 19/1, p. 357-381.

\section{MIGNON J.-M.}

1996a : «Approche morphologique et fonctionnelle de la maison: le lotissement augustéen de Saint-Florent à Orange ", in BORGARD P. (DIR.), La Maison urbaine d'époque romaine en Gaule Narbonnaise et dans les provinces voisines, Actes du colloque d'Avignon, 11-12 nov. 1994, t. I, Avignon, Association pour la promotion de la recherche archéologique en Vaucluse (coll. Documents d'archéologie vauclusienne, 6), p. 219-233.

1996b : "Orange (Vaucluse)", in BORGARD P. (DIR.), La Maison urbaine d'époque romaine en Gaule Narbonnaise et dans les provinces voisines, Atlas des maison. de Gaule Narbonnaise, t. II, Avignon, Association pour la promotion de la recherche archéologique en Vaucluse (coll. Documents d'archéologie vauclusienne, 6), p. 229-277.

\section{MiLLETT M.}

1982: «Distinguishing between the Pes 
Monetalis and the Pes Drusianus: some problems », Britannia, 13, p. 315-320.

\section{PRÉVOT P.}

2004a: Le Mobilier de tabletterie du site de la R.H.I. Saint-Florent à Orange (Vaucluse): os, ivoire, bois de cerf et bois, Mémoire de maîtrise, Université de Provence, AixMarseille-I, 328 p.

2004b : « Normalisation du travail en tabletterie gallo-romaine », Instrumentum, 20, p. 24-26.

2005 : «Les éléments de charnières en os et la question de l'excentration des goupilles latérales ", Instrumentum, 22, p. 19-21.

2006: L'Atelier de tabletterie de la colline SaintEutrope à Orange (Vaucluse, F.) : problématiques et analyses du travail de l'os, Mémoire de Master II, Université de Provence, AixMarseille-I, 2 vol., 210 p. et 167 pl.
2008: «État des connaissances sur la production de l'os à Orange (Vaucluse, F.): étude et comparaison des ateliers du travail de l'os ", in BERTRAND I. (DIR.), Le Travail de l'os, du bois de cerf et de la corne à l'époque romaine : un artisanat en marge?, Montagnac, Monique Mergoil (coll. Monographies Instrumentum, $34)$, p. $195-229$

RotTläNDER R. C. A.

1972 : « Ein angeblich spätrömische Bronze », Archäologisches Korrespondenzblatt, 2, p. 233-234.

1991: «Das X-Motiv: Ornament oder Merkmal ? ", Kölner Jahrbuch für Vor- und Frühgeschichte, 12, p. 94-101.

1994 : «Ein römischer Maßstab mit seltenen Maßeinheiten im Regionalmuseum Xanten ", Xantener Berichte: Grabung,
Forschung, Präsentation, Bd. 5, Köln, Philipp von Zabern Verlag, p. 219-227.

\section{ROUMEGOUS A.}

2003 : Étude du mobilier céramique de deux maisons augustéennes du site de Saint-Florent (B1 et E1) à Orange, Mémoire de maîtrise, Université de Provence, Aix-Marseille-I, 95 p.

\section{SCHÄRLIG A.}

2003 : « Un bas-relief à Trèves : ces Romains calculent, ils ne jouent pas!", Antike Kunst, 47, p. 65-71.

\section{ZIMMER G.}

1982: Römische Berufsdarstellungen, Berlin, Gebr. Mann Verlag (coll. Archäologische Forschungen, 12), $254 \mathrm{p}$. 